\title{
An axis-free overset grid in spherical polar coordinates for simulating 3D self-gravitating flows
}

\author{
A. Wongwathanarat, N. J. Hammer ${ }^{\star}$, and E. Müller \\ Max-Planck Institut für Astrophysik, Karl-Schwarzschild-Straße 1, 85740 Garching, Germany \\ e-mail: emueller@mpa-garching.mpg.de
}

Received 9 October 2009 / Accepted 3 March 2010

ABSTRACT

\begin{abstract}
Aims. Three dimensional explicit hydrodynamic codes based on spherical polar coordinates using a single spherical polar grid suffer from a severe restriction of the time step size due to the convergence of grid lines near the poles of the coordinate system. More importantly, numerical artifacts are encountered at the symmetry axis of the grid where boundary conditions have to be imposed that flaw the flow near the axis. The first problem can be eased and the second one avoided by applying an overlapping grid technique. Methods. A type of overlapping grid in spherical coordinates is adopted. This so called "Yin-Yang" grid is a two-patch overset grid proposed by Kageyama and Sato for geophysical simulations. Its two grid patches contain only the low-latitude regions of the usual spherical polar grid and are combined together in a simple manner. This property of the Yin-Yang grid greatly simplifies its implementation into a 3D code already employing spherical polar coordinates. It further allows for a much larger time step in $3 \mathrm{D}$ simulations using high angular resolution $\left(\lesssim 1^{\circ}\right)$ than that required in $3 \mathrm{D}$ simulations using a regular spherical grid with the same angular resolution.

Results. The Yin-Yang grid is successfully implemented into a 3D version of the explicit Eulerian grid-based code PROMETHEUS including self-gravity. The modified code successfully passed several standard hydrodynamic tests producing results which are in very good agreement with analytic solutions. Moreover, the solutions obtained with the Yin-Yang grid exhibit no peculiar behaviour at the boundary between the two grid patches. The code has also been successfully used to model astrophysically relevant situations, namely equilibrium polytropes, a Taylor-Sedov explosion, and Rayleigh-Taylor instabilities. According to our results, the usage of the Yin-Yang grid greatly enhances the suitability and efficiency of 3D explicit Eulerian codes based on spherical polar coordinates for astrophysical flows.
\end{abstract}

Key words. methods: numerical - hydrodynamics - gravitation - supernovae: general

\section{Introduction}

Three dimensional hydrodynamic simulations employing a single spherical polar grid are computationally expensive because of the convergence of grid lines towards the north and south pole. The converging grid lines imply a severe restriction of the time step size for any hydrodynamic code using explicit time discretization due to the CFL condition. This so-called "pole problem" bothers astrophysicists when simulating self-gravitating flow in three dimensions (e.g., convection in stars, or stellar explosions) where the spherical coordinate system is often preferable. In particular, simulations of core-collapse supernovae are a problem with which astrophysicists have been struggling. While observations show clear evidence of asymmetric (3D) complex structures in supernova ejecta, numerical simulations, in most cases, are carried out only in two spatial dimensions assuming axisymmetry (e.g., Blondin \& Mezzacappa 2006; Scheck et al. 2006; Ohnishi et al. 2007). Three dimensional core-collapse supernova simulations are rare (e.g., Janka et al. 2005; Mezzacappa et al. 2006; Scheck 2006; Iwakami et al. 2008). In addition to the severe restriction of the time step size, boundary conditions that have to be imposed at the symmetry axis $\theta \in[0, \pi]$ flaw the simulations near the axis by causing undesired numerical artifacts in 2D axisymmetric simulations, as e.g., jet-like flow features

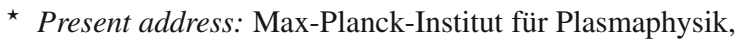
Boltzmannstraße 2, 85748 Garching, Germany.
(Kifonidis et al. 2003). In 3D simulations, the axis represents a coordinate singularity that almost unavoidably will leave its mark on the flow near or across the axis.

There have been attempts to construct a new type of grid which is able to ease the pole problem. However, it is not possible to construct a single grid patch that can cover the entire surface of a sphere, is orthogonal, and at the same time does not contain any coordinate singularity except at the origin. Therefore, multi-patch grid and overlapping (or overset) grid approaches are employed. They are widely used in the field of computational fluid dynamics where complex grid structures are common. For flows possessing an approximate global spherical symmetry, the "cubed sphere" grid (Ronchi et al. 1996) has been developed and is currently applied to several astrophysical problems (e.g. Koldoba et al. 2002; Romanova et al. 2003; Zink et al. 2008; Fragile et al. 2009). It is an overset grid consisting of six identical patches covering a solid angle of $4 \pi$ steradians. The "Yin-Yang" grid has the latter property, too, but up to now it has not been used in astrophysical applications.

The Yin-Yang grid was introduced by Kageyama \& Sato (2004). It consists of two overlapping grid patches named "Yin" and "Yang" grid. In comparison with other types of overset grids in spherical geometry, the Yin-Yang grid geometry is simple, as both the Yin and the Yang grid consist of a part of a usual spherical polar grid. The transformation of coordinates and vector components between the two patches is straightforward 
and symmetric, thus allowing for an easy and straightforward implementation of the grid into a 3D code already employing spherical polar coordinates. The Yin-Yang grid is successfully used on massively parallel supercomputers in the field of geophysical science for simulations of mantle convection and the geodynamo. In these applications the thermal convection equation and the magnetohydrodynamic (MHD) equations are solved on the Yin-Yang grid using a second-order accurate finite difference method. Here, we also adopt the Yin-Yang grid, and use it for astrophysically relevant (finite-volume) hydrodynamic simulations for the first time.

The paper is structured as follows. In Sect. 2, we describe the basics of the Yin-Yang grid configuration including the transformations of coordinates and vectors between the Yin and Yang grid patches. In Sect. 3, we provide the details of the implementation of the Yin-Yang grid into the PROMETHEUS hydrodynamic code, and also discuss the resulting necessary modifications of its 3D gravity solver that is based on spherical harmonics. In Sect. 4, we present the results of the test calculations we have performed including a test with self-gravity. In Sect. 5, we discuss the conservation problem arising when applying the YinYang grid. Then we report on the efficiency and performance gain obtained with the Yin-Yang grid compared to a spherical polar grid in Sect. 6. Finally, we give the conclusions from our study in Sect. 7.

\section{Yin-Yang grid}

The Yin-Yang grid configuration is shown in Fig. 1. Both the Yin and the Yang grid are simply a part of a usual spherical polar grid and are identical in geometry. The angular domain of each grid patch is given by

$\theta=\left[\frac{\pi}{4}-\delta, \frac{3 \pi}{4}+\delta\right] \cap \phi=\left[-\frac{3 \pi}{4}-\delta, \frac{3 \pi}{4}+\delta\right]$

where $\theta$ and $\phi$ are the colatitude and azimuth, respectively. Note that it is necessary to add at least one extra buffer grid zone to both angular directions in order to ensure an appropriate overlap of the grids. The angular width $\delta$ of this buffer zone depends on the grid resolution, i.e., $\delta \equiv \Delta \theta=\Delta \phi$, where for simplicity we assumed equal angular spacing in $\theta$ - and $\phi$-direction. The angular domain is hereby extended by $2 \delta$ in both angular directions. The Yin and Yang grid are patched together in a specific manner forming a spherical shell with a small overlapping region covering approximately $6 \%$ of a sphere's surface. Stacking up Yin-Yang shells in radial direction results in a 3D grid that is identical to the usual spherical polar grid in radial direction. It is obvious that, unlike in the case of the spherical polar grid, the problematic high latitude sections of the sphere are avoided, and the angular zoning is almost equidistant.

The Cartesian coordinates

$$
\left(x^{(n)}, y^{(n)}, z^{(n)}\right)=\left(r \sin \theta^{(n)} \cos \phi^{(n)}, r \sin \theta^{(n)} \sin \phi^{(n)}, r \cos \theta^{(n)}\right)
$$

corresponding to the Yin grid, denoted by a superscript ( $n$ ), and the Cartesian coordinates

$$
\left(x^{(e)}, y^{(e)}, z^{(e)}\right)=\left(r \sin \theta^{(e)} \cos \phi^{(e)}, r \sin \theta^{(e)} \sin \phi^{(e)}, r \cos \theta^{(e)}\right)
$$

corresponding to the Yang grid, denoted by a superscript $(e)$, are related to each other through the transformation

$$
\left(\begin{array}{l}
x^{(e)} \\
y^{(e)} \\
z^{(e)}
\end{array}\right)=M\left(\begin{array}{l}
x^{(n)} \\
y^{(n)} \\
z^{(n)}
\end{array}\right)
$$

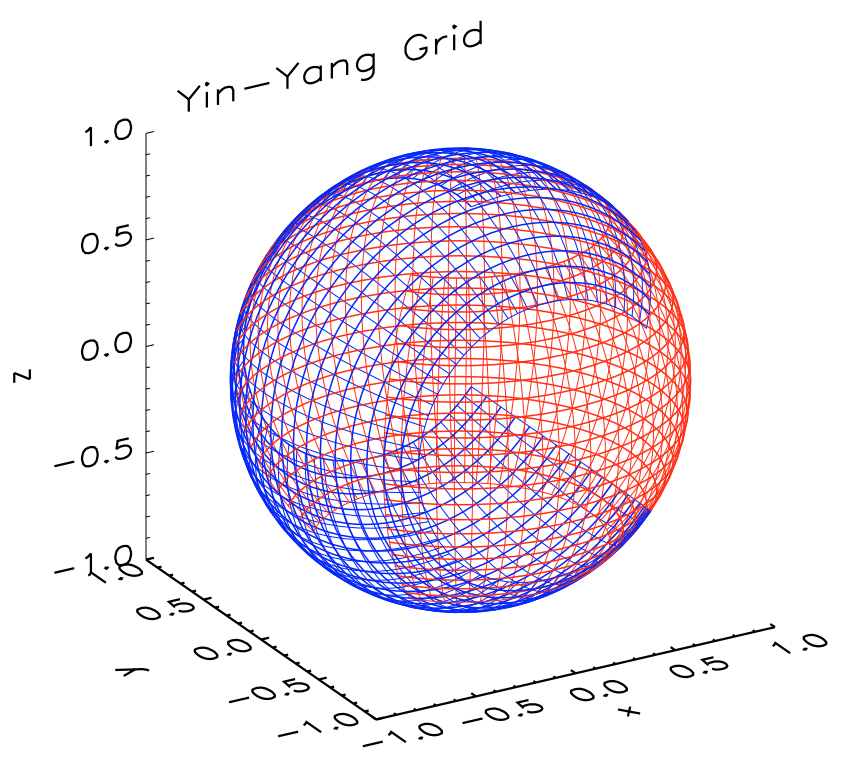

Fig. 1. An axis-free Yin-Yang grid configuration plotted on a spherical surface. Both the Yin (red) and Yang (blue) grid are the low latitude part of the normal spherical polar grid and are identical in geometry. The Yang grid is obtained from the Yin grid by two rotations, and vice versa.

where

$M=\left(\begin{array}{ccc}-1 & 0 & 0 \\ 0 & 0 & 1 \\ 0 & 1 & 0\end{array}\right)$

This Yin-Yang coordinate transformation can also be considered as two subsequent rotations. Accordingly, the transformation matrix $M$ can be written as $R_{x}\left(90^{\circ}\right) R_{z}\left(180^{\circ}\right)$, where $R_{x}$ and $R_{z}$ are the transformation matrices of rotations by $90^{\circ}$ around the $x$-axis and by $180^{\circ}$ around the $z$-axis in counterclockwise direction, respectively. For the inverse transformation matrix $M^{-1}=M$ holds.

The relation between the spherical coordinates of the Yin and Yang grid patches can be derived directly from the transformation matrix $M$. Because the Yin-Yang coordinate transformation involves only rotations, it implies that the radial coordinate is identical on the Yin and the Yang grid. The angular coordinates transform as

$$
\begin{aligned}
\theta^{(e)} & =\arccos \left(\sin \theta^{(n)} \sin \phi^{(n)}\right), \\
\phi^{(e)} & =\arctan \left(\frac{\cos \theta^{(n)}}{-\sin \theta^{(n)} \cos \phi^{(n)}}\right) .
\end{aligned}
$$

Note that the inverse transformation has the same form as (6) and (7) but exchanging the (grid) superscripts.

Vector components in spherical coordinates transform according to

$$
\left(\begin{array}{c}
v_{r}^{(e)} \\
v_{\theta}^{(e)} \\
v_{\phi}^{(e)}
\end{array}\right)=P\left(\begin{array}{c}
v_{r}^{(n)} \\
v_{\theta}^{(n)} \\
v_{\phi}^{(n)}
\end{array}\right)
$$

where

$$
P=\left(\begin{array}{ccc}
1 & 0 & 0 \\
0 & -\sin \phi^{(e)} \sin \phi^{(n)} & -\cos \phi^{(n)} / \sin \theta^{(e)} \\
0 & \cos \phi^{(n)} / \sin \theta^{(e)} & -\sin \phi^{(e)} \sin \phi^{(n)}
\end{array}\right)
$$


is the vector transformation matrix. When switching (grid) superscripts $(e)$ and $(n)$ in matrix $P$, the inverse vector transformation matrix is obtained. For a detailed derivation of the transformation matrix $P$, we refer to Sect. 3 of Kageyama \& Sato (2004). Note that the vector transformation matrix $P$ is singular at $\sin \theta^{(e)}=0$, but this singular point is rectifiable. In practice, one can always decompose vectors into their Cartesian components and perform the corresponding transformation.

\section{Implementation}

We have implemented the Yin-Yang grid into our explicit finite-volume Eulerian hydrodynamics code, PROMETHEUS, which integrates the equations of multidimensional hydrodynamics using the piecewise parabolic method (PPM; Collela \& Woodward 1984) and dimensional splitting. The code also includes a Poisson solver based on spherical harmonics to handle self-gravity.

\subsection{Hydrodynamics solver}

Firstly, the Yin-Yang grid needs to be constructed. Since both the Yin and the Yang grid are part of a spherical polar grid an analogous spatial discretization in angular direction can be used. For example, the $\theta$ and $\phi$ coordinates of the zone center of an angular zone $(j, k)$ of a Yin-Yang grid, having $N_{\theta}$ zones in $\theta$-direction and $N_{\phi}$ zones in $\phi$-direction, are given by

$$
\begin{array}{cc}
\theta_{j}=\theta_{\min }+j \Delta \theta-\frac{\Delta \theta}{2} & \text { for } \quad 1 \leq j \leq N_{\theta}, \\
\phi_{k}=\phi_{\min }+k \Delta \phi-\frac{\Delta \phi}{2} & \text { for } \quad 1 \leq k \leq N_{\phi},
\end{array}
$$

where

$$
\begin{aligned}
\Delta \theta & =\frac{\theta_{\max }-\theta_{\min }}{N_{\theta}}, \\
\Delta \phi & =\frac{\phi_{\max }-\phi_{\min }}{N_{\phi}}
\end{aligned}
$$

are the respective angular grid spacings.

The range of values for the colatitude $\theta$ and the azimuth angle $\phi$ are as given in (1), and for simplicity we set $\Delta \theta=\Delta \phi$. In radial direction no modification is required. The geometric property of the Yin-Yang grid allows us to make use of the coordinate arrays $r_{i}, \theta_{j}$, and $\phi_{k}$ twice by enforcing the same grid resolution for both grid patches. This approach avoids doubling the coordinate arrays.

Only simple modifications are needed concerning the data and program structure. Arrays with three spatial indices, e.g., $i$, $j$, and $k$, need an extra grid index, say, $l$. For example, the array for the density field will be $\rho(i, j, k, l)$ instead of $\rho(i, j, k)$. As a consequence any triple loop running over indices $i, j$, and $k$ in the program becomes a fourfold loop over $i, j, k$, and $l$ instead. Otherwise, the Yin-Yang grid allows one to exploit without any further modification any already implemented finitevolume scheme in spherical coordinates to solve the equations of hydrodynamics.

Different from the spherical polar grid, the Yin-Yang grid requires no boundary conditions in angular directions. Each grid patch communicates with its neighboring patch using information from ghost zones that is obtained by interpolation of data between internal grid zones of the neighboring grid patch. Interpolation is only required in the two angular coordinates

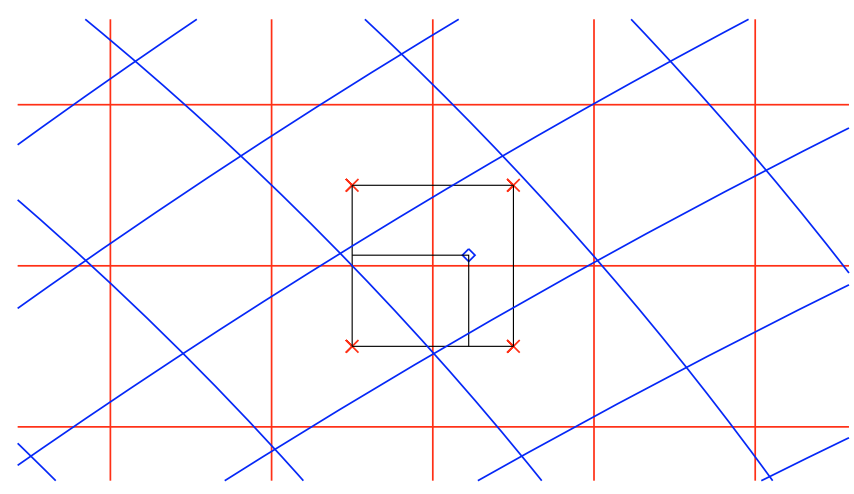

Fig. 2. A Mercator projection of an overlap region of the Yin-Yang grid. In case of bi-linear interpolation, four neighboring values of the underlying grid (red) will be used to determine the zone-centered value of a ghost zone in the grid on top (blue). The interpolation coefficients are determined by the relative distances, denoted by black lines, between the interpolation point (diamond) and the four neighboring points (crosses).

as the radial part of the Yin-Yang grid is identical to that of a spherical polar grid. It is straightforward to determine the corresponding interpolation coefficients. The mapping of vector quantities between the Yin and Yang grid patches requires an additional step. After interpolating the vector components they must be transformed according to the transformation given in Eq. (8) from the Yin to the Yang angular coordinate system, and vice versa.

We tested two interpolation procedures. In the first one all primitive state variables (density, velocity, energy, pressure, temperature, abundances) are interpolated ignoring the resulting small thermodynamic inconsistencies. In the second procedure, we only interpolate the conserved quantities (density, momentum, total energy, and abundances), and compute the velocity and the remaining thermodynamic state variables consistently via the equation of state. Both procedures produce very similar results which differ at the level of the discretization errors. As the second procedure is more consistent we use it as the standard one in our code.

An example of overlapping situations which are encountered when using a Yin-Yang grid is shown in Fig. 2. For simplicity, we use bi-linear interpolation in order to prevent unwanted oscillation. Because the grid patches are fixed in both angular directions the interpolation coefficients for each ghost zone need to be calculated only once per simulation at the initialization step. After initialization, the coefficient map is stored in an array for later usage. Moreover, the symmetry property of the Yin-Yang transformation allows one to make use of the interpolation coefficients twice for both grids.

Because the Yin-Yang grid is an overlapping grid integral quantities such as the total mass or total energy on the computational domain cannot be obtained by just summing local quantities from every grid cells. Doing so will result in counting the contributions in the overlapping region twice. To circumvent this problem, weights are given to each grid zone during the summation. Suppose a grid zone has an overlapping volume fraction $\alpha$ the cell will receive a weight $w=1.0-0.5 \alpha$. Zones in the nonoverlapping region receive the weight of 1.0, i.e., the entire zone contributes to the integral while, on the other hand, zones that are fully contained within the overlapping region have a weight 0.5 . The volume fraction $\alpha$ does not depend on the radial coordinate and can be thought of as an area fraction since the grid patches 
are not offset in radial direction. Prior to the area integration, one needs to determine for each zone interface of the underlying grid the points where the interface is intersected by the boundary lines of the other grid, e.g., points on the Yin grid intersected by the boundary lines of the Yang grid. The intersection points can be determined using the Yin-Yang coordinate transformation in (6) and (7), respectively. The integration in the overlapping area is then carried out using the trapezoidal method. This procedure is also described in Peng et al. (2006). Once the area or volume fraction $\alpha$ is calculated, the weights for each cell are obtained easily. Note that these weights need to be calculated only at the initialization step, and are stored for later usage in a coefficient map $w(j, k)$, where $j$ and $k$ are the indices referring to the $\theta$ and $\phi$ coordinates, respectively. The coefficient map can be applied to both grids without any modification. Using the above described approach, the volume or surface area of the grids can be calculated with an accuracy up to machine precision.

\subsection{Gravity solver}

The 3D Newtonian gravitational potential is computed from Poisson's equation in its integral form using an expansion into spherical harmonics as described in Müller \& Steinmetz (1995). Because the algorithm of these authors is based on a (single) spherical polar grid the density on the Yin-Yang sphere has to be interpolated onto an auxiliary spherical polar grid. The interpolation used is first-order accurate, and due to the simplicity of the Yin-Yang grid configuration has to be performed only in the two angular dimensions. Concerning the resolution of the auxiliary grid, it is natural to employ the same grid resolution as that used for the Yin-Yang grid in all three spatial dimensions. The orientation of the auxiliary grid can be chosen freely in principle. However, it is convenient to align it with one of the two grid patches (the Yin-grid in our case). Once the density is interpolated onto the auxiliary spherical grid we compute the gravitational potential, as suggested by Müller \& Steinmetz (1995), at zone interfaces instead of at zone centers on both the Yin and Yang grid. The gravitational acceleration at zone centers can then be obtained by central differencing the potential. Note that the interpolation coefficients for the density need to be calculated only once per simulation, because both the auxiliary grid and the Yin-Yang grid are fixed in angular directions. In addition, all angular weights, Legendre polynomials, and their integrals required for the calculation of the gravitational potential are stored after the initialization step for later usage.

It is also possible to directly calculate the gravitational acceleration at zone centers. The gravitational potential is given by (see Eqs. (5)-(7) in Müller \& Steinmetz 1995)

$$
\begin{aligned}
\Phi(r, \theta, \phi)= & -G \sum_{l=0}^{\infty} \frac{4 \pi}{2 l+1} \\
& \times \sum_{m=-l}^{l} Y^{l m}(\theta, \phi)\left(\frac{1}{r^{l+1}} C^{l m}(r)+r^{l} D^{l m}(r)\right)
\end{aligned}
$$

with

$$
\begin{aligned}
& C^{l m}(r)=\int_{4 \pi} \mathrm{d} \Omega^{\prime} Y^{l m *}\left(\theta^{\prime}, \phi^{\prime}\right) \int_{0}^{r} \mathrm{~d} r^{\prime} r^{\prime l+2} \rho\left(r^{\prime}, \theta^{\prime}, \phi^{\prime}\right), \\
& D^{l m}(r)=\int_{4 \pi} \mathrm{d} \Omega^{\prime} Y^{l m *}\left(\theta^{\prime}, \phi^{\prime}\right) \int_{r}^{\infty} \mathrm{d} r^{\prime} r^{\prime 1-l} \rho\left(r^{\prime}, \theta^{\prime}, \phi^{\prime}\right),
\end{aligned}
$$

where $Y^{l m}$ and $Y^{l m *}$ are the spherical harmonics and their complex conjugates, $\rho$ is the density, and $\mathrm{d} \Omega \equiv \sin \theta \mathrm{d} \theta \mathrm{d} \phi$. The gravitational acceleration in radial direction is then

$$
\begin{aligned}
& \frac{\partial}{\partial r} \Phi(r, \theta, \phi)= \\
& -G \sum_{l=0}^{\infty} \frac{4 \pi}{2 l+1} \sum_{m=-l}^{l} Y^{l m}(\theta, \phi) \frac{\mathrm{d}}{\mathrm{d} r}\left(\frac{1}{r^{l+1}} C^{l m}(r)+r^{l} D^{l m}(r)\right) .
\end{aligned}
$$

Writing the radial derivative in Eq. (17) as

$$
\begin{aligned}
& \frac{\mathrm{d}}{\mathrm{d} r}\left(\frac{1}{r^{l+1}} C^{l m}(r)+r^{l} D^{l m}(r)\right)=\frac{1}{r^{l+1}} \frac{\mathrm{d}}{\mathrm{d} r} C^{l m}(r)-\frac{l+1}{r} \cdot \frac{1}{r^{l+1}} C^{l m}(r) \\
& \quad+r^{l} \frac{\mathrm{d}}{\mathrm{d} r} D^{l m}(r)+\frac{l}{r} \cdot r^{l} D^{l m}(r),
\end{aligned}
$$

and noticing that the first and third term on the right hand side of this expression cancel each other because of the identities

$$
\frac{\mathrm{d}}{\mathrm{d} x} \int_{0}^{x} f\left(x^{\prime}\right) \mathrm{d} x^{\prime}=f(x)
$$

and

$$
\frac{\mathrm{d}}{\mathrm{d} x} \int_{x}^{\infty} f\left(x^{\prime}\right) \mathrm{d} x^{\prime}=-f(x)
$$

the gravitational acceleration in radial direction becomes

$$
\begin{aligned}
\frac{\partial}{\partial r} \Phi(r, \theta, \phi)= & -G \sum_{l=0}^{\infty} \frac{4 \pi}{2 l+1} \sum_{m=-l}^{l} Y^{l m}(\theta, \phi) \\
& \times\left(-\frac{l+1}{r} \cdot \frac{1}{r^{l+1}} C^{l m}(r)+\frac{l}{r} \cdot r^{l} D^{l m}(r)\right) .
\end{aligned}
$$

The corresponding expressions for the gravitational acceleration in the two angular directions are easy to obtain since the spherical harmonics $Y^{l m}$ are the only angular-dependent terms in Eq. (14). Therefore, we only need to consider the partial derivatives of the spherical harmonics with respect to the $\theta$ and $\phi$ coordinates. As the spherical harmonics are given by

$Y^{l m}(\theta, \phi)=N^{l m} P^{l m}(\cos \theta) \mathrm{e}^{\mathrm{i} m \phi}$,

where $N^{l m}$ is the normalization constant and $P^{l m}$ the associated Legendre polynomial, one finds

$\frac{\partial}{\partial \theta} Y^{l m}(\theta, \phi)=N^{l m} \mathrm{e}^{\mathrm{i} m \phi} \frac{\mathrm{d}}{\mathrm{d} \theta} P^{l m}(\cos \theta)$

and

$\frac{\partial}{\partial \phi} Y^{l m}(\theta, \phi)=\mathrm{i} m Y^{l m}(\theta, \phi)$.

The derivatives of the associated Legendre polynomials are easily obtained using the recurrence formula

$\left(x^{2}-1\right) \frac{\mathrm{d}}{\mathrm{d} x} P_{l}^{m}(x)=l x P_{l}^{m}(x)-(l+m) P_{l-1}^{m}(x)$.

Thus, one finds for the gravitational acceleration in the angular directions

$$
\begin{aligned}
\frac{1}{r} \frac{\partial}{\partial \theta} \Phi(r, \theta, \phi)= & -\frac{G}{r} \sum_{l=0}^{\infty} \frac{4 \pi}{2 l+1} \sum_{m=-l}^{l} N^{l m} \mathrm{e}^{\mathrm{i} m \phi} \frac{\mathrm{d}}{\mathrm{d} \theta} P^{l m}(\cos \theta) \\
& \times\left(\frac{1}{r^{l+1}} C^{l m}(r)+r^{l} D^{l m}(r)\right)
\end{aligned}
$$


and

$$
\begin{aligned}
\frac{1}{r \sin \theta} \frac{\partial}{\partial \phi} \Phi(r, \theta, \phi)= & -\frac{G}{r \sin \theta} \sum_{l=0}^{\infty} \frac{4 \pi}{2 l+1} \sum_{m=-l}^{l} \mathrm{i} m Y^{l m}(\theta, \phi) \\
& \times\left(\frac{1}{r^{l+1}} C^{l m}(r)+r^{l} D^{l m}(r)\right) .
\end{aligned}
$$

Obviously, the expressions for three components of the gravitational acceleration (see Eqs. (21), (26), and (27)) are similar to that for the gravitational potential itself (see Eq. (14)). Hence, besides computing derivatives of Legendre polynomials, our extended Poisson solver can provide without much additional effort both the gravitational potential and the corresponding acceleration.

Usage of the analytic expressions for the gravitational acceleration avoids the errors arising from the numerical differentiation of the gravitational potential. However, tests show that the results obtained using either the gravitational potential computed with the "standard" Poisson solver and subsequent numerical differentiation or directly the gravitational acceleration provided by the extended Poisson solver differ only very slightly (see next section). Thus, we decided to stick to the "standard" Poisson solver in our simulations and compute the gravitational acceleration by numerical differentiation, as it requires no modification of our code.

\section{Test suites}

\subsection{Sod shock tube}

The first problem of our test suite is the planar Sod shock tube problem, a classical hydrodynamic test problem (Sod 1978). We simulated this (1D Cartesian) flow problem using spherical coordinates and the Yin-Yang grid. The initial state consists of two constant states given by

$\left(\rho, p, v_{x}\right)=\left\{\begin{array}{ll}(1.0,1.0,0.0) & \text { if } x^{(n)}>0.4 \mathrm{~cm} \\ (0.125,0.1,0.0) & \text { if } x^{(n)} \leq 0.4 \mathrm{~cm}\end{array}\right.$,

where $\rho, p$, and $v_{x}$ are the density, pressure and the velocity in $x$-direction of the fluid, respectively. We assume the fluid to obey an ideal gas equation of state with an adiabatic index $\gamma=1.4$. The surface separating the two constant states is a plane orthogonal to the $x$-axis located at $x^{(n)}=0.4 \mathrm{~cm}$, the (positive) $x$-axis corresponding to a radial ray with angular coordinates $\theta^{(n)}=\pi / 2$ and $\phi^{(n)}=0$. Thus, this 1D planar Sod shock tube problem invokes all three spherical velocity components $v_{r}, v_{\theta}$, and $v_{\phi}$ when simulating the flow in spherical polar coordinates. This allows us to test both the scalar and vector transformations as well as the interpolation between the Yin and Yang grid patches. The simulation was carried out on an equidistant Yin-Yang grid of $400(r) \times 92(\theta) \times 272(\phi) \times 2$ zones (i.e., with an angular resolution of one degree; see Eq. (1)). In radial direction the computational domain ranges from $r=0.05 \mathrm{~cm}$ to $r=1.0 \mathrm{~cm}$. We impose a zero-gradient boundary condition at both edges of the radial domain.

The solution of the shock tube problem is well-known. We compare our results with the solution calculated using an exact Riemann solver (Toro 1997). For comparison, data are resampled along the $x$-direction with a spacing $\Delta x=0.002 \mathrm{~cm}$. Figure 3 shows one dimensional profiles of $\rho, p, v_{x}$, and $e$ (specific internal energy), respectively, along the $x$-direction at $z^{(n)}=0.25 \mathrm{~cm}$ and $y^{(n)}=0 \mathrm{~cm}$ (dashed-dotted line in Fig. 4)

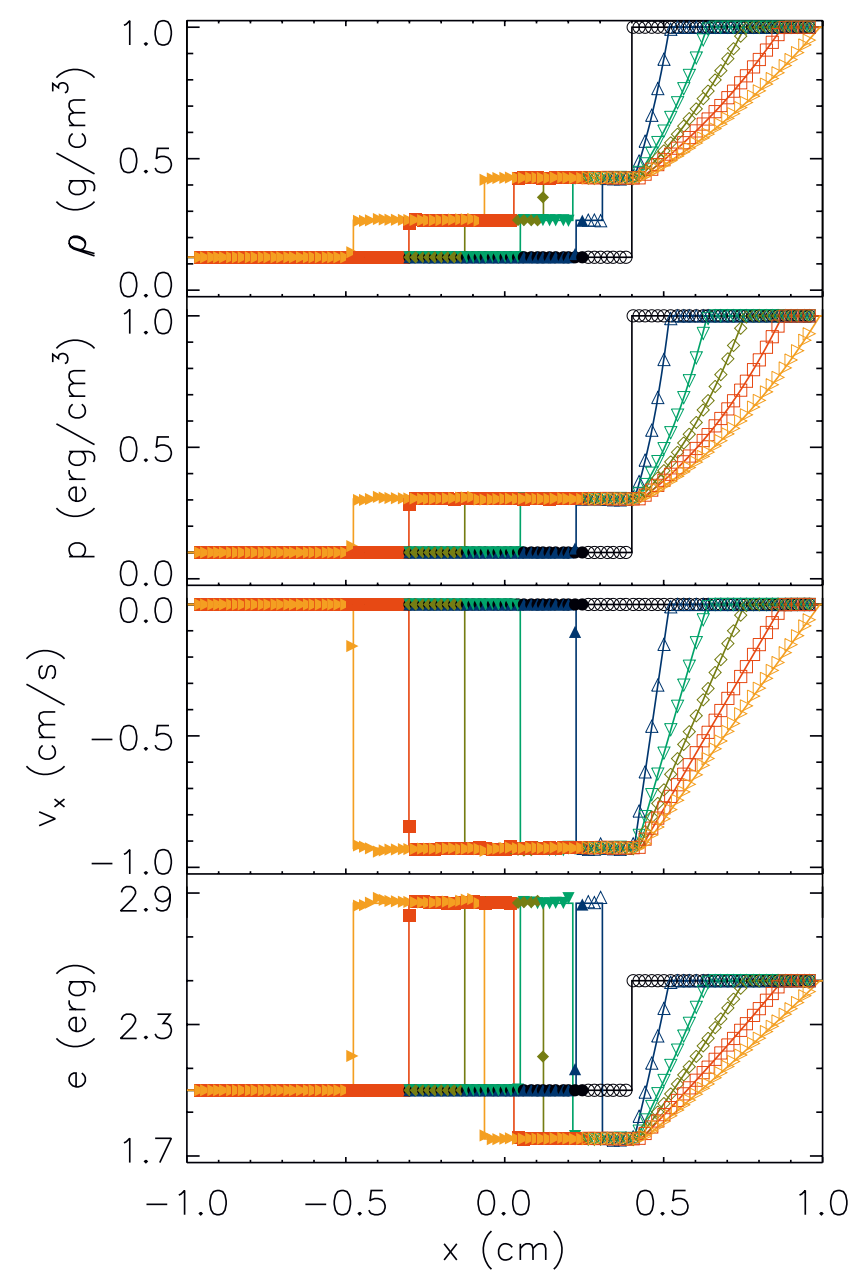

Fig. 3. One dimensional profiles of density $\rho$, pressure $p$, velocity in $x$-direction $v_{x}$, and specific internal energy $e$ are shown along the $x$-direction at $z^{(n)}=0.25 \mathrm{~cm}$ and $y^{(n)}=0 \mathrm{~cm}$ (dashed-dotted line in Fig. 4) for the shock tube simulation at every $0.1 \mathrm{~s}$. Open and filled symbols represent data points on the Yin and Yang grid, respectively. Solid lines give the distributions calculated with an exact Riemann solver.

at different times. Our results agree very well with the solution obtained with the exact Riemann solver. The grid resolution is sufficiently high to give a sharp shock front and contact discontinuity while the rarefaction wave is smooth. The shock position is correct at all time throughout the simulation. The resampled data yield an accuracy of approximately $6 \%$ on average for shock positions. The shock wave and the contact discontinuity propagate smoothly across the Yin-Yang boundary located at $x^{(n)}=0.25 \mathrm{~cm}$ without any noticeable effect by the existence of the boundary. To illustrate this behavior, Fig. 4 shows lines of constant density in the meridional plane $\phi^{(n)}=0$ at time $t=0.15 \mathrm{~s}$. The isocontours are nearly perfectly straight lines perpendicular to the $x$-axis that are unaffected by the Yin-Yang boundary (dashed line). The contour lines are slightly bent near the outer radial edge of the computational domain due to the zero-gradient boundary condition we have imposed there.

In order to firmly demonstrate that the Yin-Yang boundary does not cause numerical artifacts, we also computed this shock tube problem with a standard spherical polar grid using the same radial and angular resolution as for the Yin-Yang grid described above, i.e., $400(r) \times 180(\theta) \times 360(\phi)$. We imposed reflecting boundary conditions in $\theta$-direction and periodic ones in $\phi$-direction. Figure 5 shows a comparison of the results obtained 


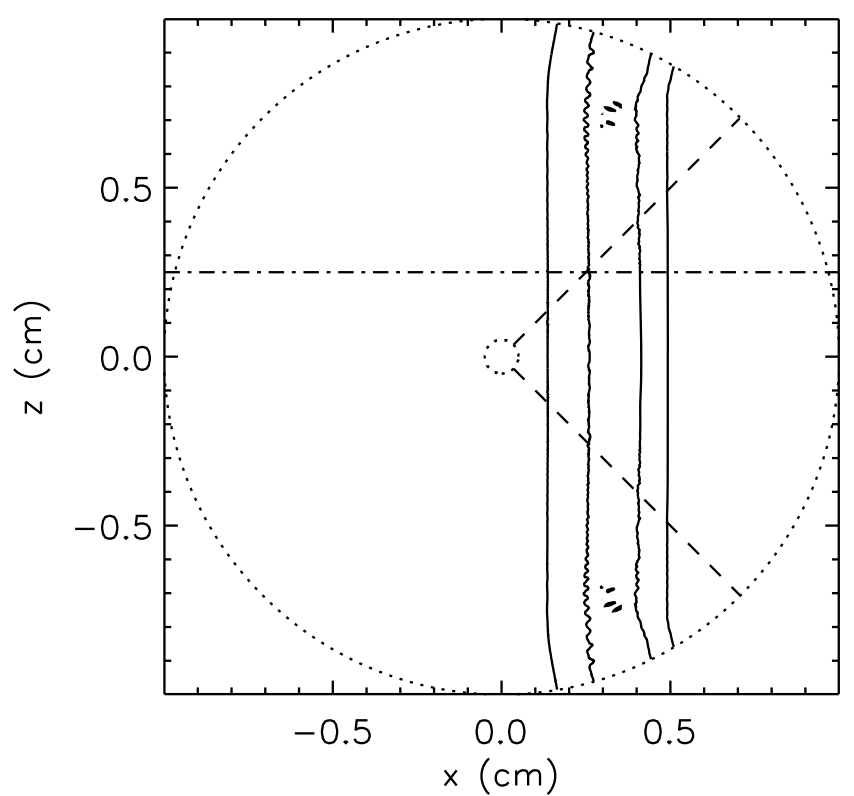

Fig. 4. Snapshot of density contours in the meridional plane $\phi^{(n)}=0$ at $t=0.15 \mathrm{~s}$ for the shock tube test problem. Dashed lines mark the YinYang grid boundary, while the dotted circular curves represent the inner and outer radial boundary of the computational domain, respectively. The one dimensional profiles shown in Fig. 3 are re-sampled along the dashed-dotted line at $z^{(n)}=0.25 \mathrm{~cm}$.

with both simulations. The two panels give the tangential velocity, defined as $\sqrt{\left(v_{y}^{(n)}\right)^{2}+\left(v_{z}^{(n)}\right)^{2}}$, in the meridional plane $\phi^{(n)}=0$ at time $t=0.15 \mathrm{~s}$ for the Yin-Yang grid (left), and the standard spherical polar grid (right), respectively. This velocity component should remain exactly zero because of the chosen initial conditions. Thus, it is a sensitive indicator whether the Yin-Yang boundary works properly, which obviously is indeed the case as the left panel of Fig. 5 shows no hint of the location of that boundary. The modulus of the tangential velocity does nowhere exceed a value of $0.05 \mathrm{~cm} / \mathrm{s}$ or approximately $5 \%$ of the shock velocity (in $x$-direction) except near the outer radial edge of the grids, where the boundary condition causes larger numerical errors. Note that nonzero tangential velocities are encountered on both the Yin-Yang grid and the standard spherical polar grid in the same grid regions at the same level. We thus conclude that they are the result of numerical errors that unavoidably occur when propagating a planar shock across a spherical polar grid, be it a standard one or a Yin-Yang grid.

\subsection{Taylor-Sedov explosion}

As a second test for our code we consider the Taylor-Sedov explosion problem. We set up the initial state for the problem by mapping a spherically symmetric analytic solution (Landau \& Lifshitz 1959) onto the computational grid. We choose the parameters of the problem to mimic a supernova explosion in an interstellar medium. Because the shock wave resulting from the explosion is spherically symmetric with respect to the center of the explosion, we assume the explosion center to be located at the point $\left(x^{(n)}, y^{(n)}, z^{(n)}\right)=(7.0,0.0,2.5) \times 10^{19} \mathrm{~cm}$. Hence, this second test problem also involves a non-zero flux of mass, momentum, and energy across the Yin-Yang boundary, and as the previous shock tube test, it probes whether that boundary causes any numerical artifacts.
The initial shock radius is $r_{0}=2.9625 \times 10^{19} \mathrm{~cm}$ orresponding to a time $t_{\exp }=0.34 \times 10^{11} \mathrm{~s}$ past the onset of the explosion, and the explosion energy was set to $E_{0}=10^{51} \mathrm{erg}$. The ambient medium into which the shock wave is propagating is at rest. It has a constant density $\rho_{\mathrm{b}}=10^{-25} \mathrm{~g} / \mathrm{cm}^{3}$, and a constant pressure $p_{\mathrm{b}}=1.4 \times 10^{-13} \mathrm{erg} / \mathrm{cm}^{3}$. The fluid is described by an ideal gas equation of state with an adiabatic index $\gamma=5 / 3$, resulting in a density jump across the shock front of $(\gamma+1) /(\gamma-1)=4$. We use a grid resolution of $400 \times 92 \times 272 \times 2$ zones, a computational domain covering the radial interval $r=[0.5,15] \times 10^{19} \mathrm{~cm}$, and employ a zero-gradient boundary condition at both the inner and the outer radial boundary.

Our results are shown together with the analytic solution in Fig. 6. We have re-sampled our data and calculated radial profiles of the density $\rho$, pressure $p$, and radial velocity $v_{r}$ along a line in $z$-direction through the explosion center using a uniform radial spacing $\Delta r=10^{18} \mathrm{~cm}$. As one can see the numerical results agree very well with the analytic solution. All flow quantities are smooth across the Yin-Yang boundary, i.e., the shock wave passes that boundary without any noticeable numerical artifact. Due to the finite resolution the density jump across the shock front is slightly smaller in the simulation than the analytic value of four. However, the shock front is sharp throughout the whole simulation, and it propagates with the correct speed. One distinct feature of the Taylor-Sedov solution is its spherical symmetry. To illustrate that the Yin-Yang grid does not destroy this symmetry of the solution, we show a set of lines of constant density in the meridional plane $\phi^{(n)}=0$ in Fig. 7. We also marked the line (dashed-dotted) along which the data given in Fig. 6 are re-sampled. The contour lines, all of which are almost perfectly circular, are drawn at a simulation time $t_{\text {sim }}=2.0 \times 10^{11} \mathrm{~s}$ (i.e., time step number 1276) corresponding to an explosion time $t_{\exp } \approx 2.34 \times 10^{11} \mathrm{~s}$.

We further studied how the solution differs in the region where the Yin and Yang grid overlap. To this end we compare the total mass within the overlap region computed on the Yin and the Yang grid, respectively. Figure 8 shows the evolution of the relative mass difference, i.e., the mass within the overlap region computed on the Yin grid, $M_{n}^{\text {ovlp }}$ minus the mass computed on the Yang grid, $M_{e}^{\text {ovlp }}$, divided by the sum of these two masses. We calculated this quantity for three different (angular) grids with $400 \times 32 \times 92 \times 2$ zones (i.e., $3^{\circ}$ angular resolution), $400 \times 92 \times 272 \times 2$ zones (i.e., $1^{\circ}$ angular resolution), and $400 \times 182 \times 542 \times 2$ (i.e., $0.5^{\circ}$ angular resolution), respectively. For all three grid resolutions the relative mass difference has a value of about $10^{-4}$. Although its evolution with time is different in case of the $3^{\circ}$ simulation (because the coarse angular grid causes large errors when mapping the analytic initial data onto the grid which determine the further evolution), Fig. 8 shows that for an angular resolution better than $1^{\circ}$ the relative mass difference behaves similarly, its maximum value decreasing from $2.1 \times 10^{-4}$ at $1^{\circ}$ angular resolution to $1.5 \times 10^{-4}$ at $0.5^{\circ}$ angular resolution.

\subsection{Rayleigh-Taylor instability}

We also simulated a single mode Rayleigh-Taylor instability (RTI) on a Yin-Yang sphere. The initial configuration consists of a spherical shell of a heavier fluid of density $\rho_{\mathrm{H}}=2 \mathrm{~g} / \mathrm{cm}^{3}$ that is supported against a constant gravitational field $g=1 \mathrm{~cm} / \mathrm{s}^{2}$ pointing in negative radial direction by a spherical shell of a lighter fluid of density $\rho_{L}=1 \mathrm{~g} / \mathrm{cm}^{3}$. The boundary between the two fluid shells is initially located at a radius $r=0.5 \mathrm{~cm}$. To 
A. Wongwathanarat et al.: An axis-free overset grid in spherical polar coordinates for simulating 3D self-gravitating flows
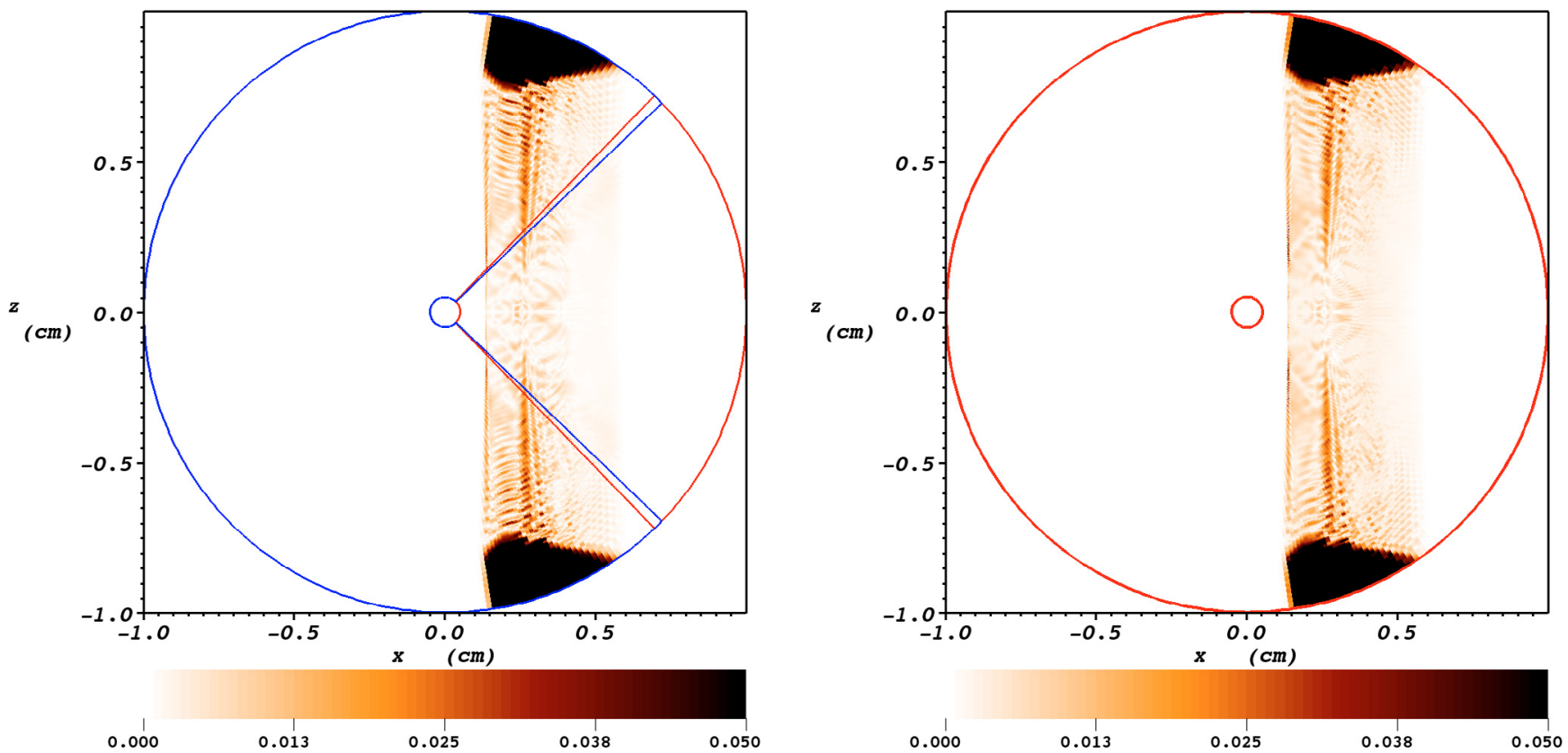

Fig. 5. Color maps of the tangential velocity defined by $\sqrt{\left(v_{y}^{(n)}\right)^{2}+\left(v_{z}^{(n)}\right)^{2}}$ in the meridional plane $\phi^{(n)}=0$ resulting from the (1D Cartesian) shock tube problem. The snapshots are computed using the Yin-Yang grid (left) and a standard spherical polar grid (right) at a time $t=0.15 \mathrm{~s}$. On the left panel, red and blue lines mark the boundaries of the Yin and the Yang grid patches, respectively. On the right panel, the two red circles show the inner and outer boundary in the radial direction of the standard spherical polar grid. The labels at the color bars give the tangential velocity in units of $\mathrm{cm} / \mathrm{s}$. The color range is limited to $0.05 \mathrm{~cm} / \mathrm{s}$ to emphasize the smallness of the tangential velocity far from the outer radial grid boundary.

balance the gravitational force, the initial (radial) pressure distribution is set to

$P(r)= \begin{cases}P_{0}+g \rho_{\mathrm{H}}(1.0-r) & \text { if } r \geq 0.5 \mathrm{~cm} \\ P(r=0.5)+g \rho_{L}(0.5-r) & \text { if } r<0.5 \mathrm{~cm}\end{cases}$

where $P_{0}=1 \mathrm{erg} / \mathrm{cm}^{3}$. A radial velocity varying in angular direction as the spherical harmonics $Y_{l}^{m}(\theta, \phi)$ with $l=3$ and $m=2$ is used to perturb the initial configuration. The amplitude of the velocity perturbation is $2.5 \%$ of the local sound speed $c_{s}(r)$. Hence, the initial radial velocity is given by

$v_{r}(r, \theta, \phi)=-0.025 \times c_{s}(r) Y_{3}^{2}(\theta, \phi)$.

The spherical harmonics $Y_{l}^{m}(\theta, \phi)$ are connected with the associated Legendre polynomials $P_{l}^{m}$ via the expression

$Y_{l}^{m}(\theta, \phi)=\sqrt{\frac{(l-m) !}{(l+m) !}} P_{l}^{m}(\cos \theta) \mathrm{e}^{\mathrm{i} m \phi}$.

The perturbation mode $(l, m)=(3,2)$ yields a maximum radial velocity in the directions

$(\theta, \phi)=\{(\pi-\alpha, 0),(\pi-\alpha, \pi),(\alpha, \pi / 2),(\alpha,-\pi / 2)\}$,

where $\alpha \equiv \arccos (\sqrt{3} / 3)$. The remaining two velocity components of the perturbation mode are set equal to 0 . The fluids are described by an ideal gas equation of state with an adiabatic index $\gamma=1.4$. The simulation is carried out on a Yin-Yang grid of $400 \times 92 \times 272 \times 2$ zones. To keep the fluid in hydrostatic equilibrium, a zero-gradient boundary condition is used for both the inner and outer boundary in radial direction. The inner radial boundary is located at $r=0.1 \mathrm{~cm}$.

A snapshot of the resulting density distribution obtained with the Yin-Yang grid is displayed in Fig. 9 at epoch $t=2.85 \mathrm{~s}$. The left panel shows color coded contour lines in $3 \mathrm{D}$, and the right one a meridional cut at $\phi^{(n)}=0$. The contour lines are drawn using different color tables for the Yin and Yang grid, respectively. Four distinct bubbles of rising low density fluid (Yin: blue; Yang: bright gray) are clearly visible that reflect the initial perturbation mode $(l, m)=(3,2)$. High density fluid (Yin: yellow/red; Yang: dark gray/black) sinks down and settles at the inner part of the sphere. One can also notice Kelvin-Helmholtz instabilities developing at the surface of the bubbles. This is particularly obvious in the meridional cut (right panel). One of the RTI bubbles is within the Yang grid, while the three others reside on the Yin grid. It is obvious that the bubbles are distributed symmetrically following the perturbation pattern regardless of the grid patch. The 2D contour lines shown in the right panel of Fig. 9 emphasize this fact.

The RTI bubbles grow with nearly the same growth rate in all four (perturbation) directions, as can also be seen from Fig. 10 that displays the position of each bubble's head versus time. The four curves lie exactly on top of each other during the phase of linear growth. There are slight discrepancies between the four curves in the non-linear regime, because the linear grid resolution in angular direction is slightly non-equidistant (due to its $\theta$ dependence). Two curves from the Yin grid coincide perfectly since they represent the two bubbles that lie symmetrically above and below the equator in the Yin grid. The results confirm that the Yin-Yang grid does not favor any angular direction on the sphere. Since our aim was only to demonstrate this important fact, we do not further analyze the growth rate of the RTI.

\subsection{Gravitational potential of homogeneous spheroids}

We investigate the accuracy of our gravity solver by calculating the gravitational potential of homogeneous spheroids. We consider two homogeneous self-gravitating configurations: a prolate spheroid with an axis ratio of 0.7 , and a sphere. The 


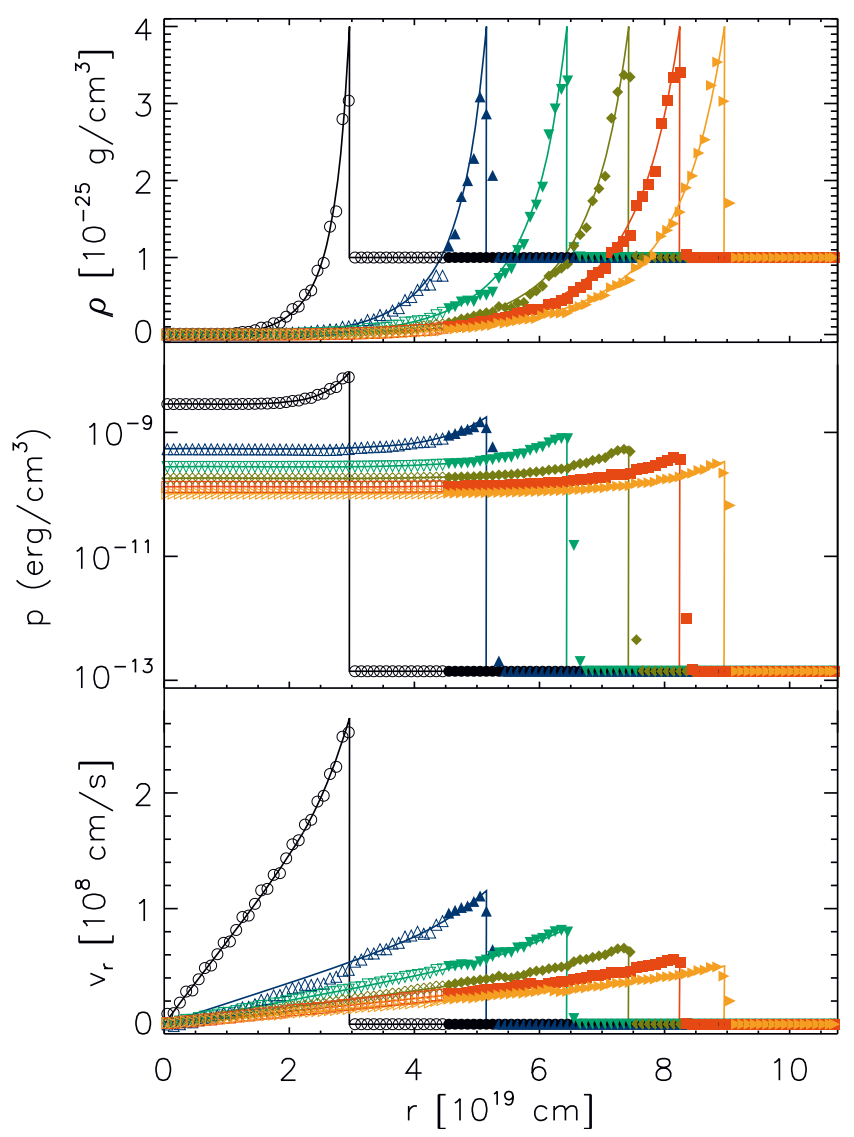

Fig. 6. Distributions of density (top), pressure (middle) and radial velocity (bottom) versus radius from the explosion center (located at $\left(x^{(n)}, y^{(n)}, z^{(n)}\right)=(7.0,0.0,2.5) \times 10^{19} \mathrm{~cm}$ for the Taylor-Sedov explosion problem plotted at every $10^{11} \mathrm{~s}$. Open symbols are data points from the Yin grid, while filled symbols represent sampled data from the Yang grid. The solid lines give the corresponding analytic solution. The data are re-sampled along the dashed-dotted line shown in Fig. 7.

configurations have a constant density $\rho=1 \mathrm{~g} / \mathrm{cm}^{3}$, and are embedded into a homogeneous background of much lower density $\rho_{\mathrm{b}}=10^{-20} \mathrm{~g} / \mathrm{cm}^{3}$ in order to minimize the background's contribution to the gravitational potential. The semi-major axis of the spheroid aligns with the $x$-axis, while its center is placed at the origin of the Yin-Yang grid. To provide a more difficult test for our multipole based gravity solver, we shift the center of the sphere off the origin of the computational grid by more than one sphere radius.

The analytical form of the gravitational potential for both type of configurations are known. The solution for the prolate spheroid can be found in Chap. 3 of Chandrasekhar (1969), and the sphere's potential can be easily calculated. Figure 11 shows contour lines of the gravitational potential for both cases in the meridional plane $\phi^{(n)}=0$. The potential is calculated on a grid of $400 \times 92 \times 272 \times 2$ zones with $L=15$, where $L$ is the number of spherical harmonics taken into account (see Sect. 3.2). The contour lines are smooth across the Yin-Yang boundary for both the prolate spheroid and the sphere.

Concerning the convergence behavior of the solver, we consider various grid resolutions and a number of spherical harmonics ranging up to $L=25$ for this convergence test. The grid resolutions used in the test are $400 \times 92 \times 272 \times 2$ zones, $400 \times 47 \times 137 \times 2$ zones, $200 \times 92 \times 272 \times 2$ zones, and $200 \times 47 \times 137 \times 2$ zones, respectively. The maximum and mean error of the gravitational potential are given as a function of $L$ for

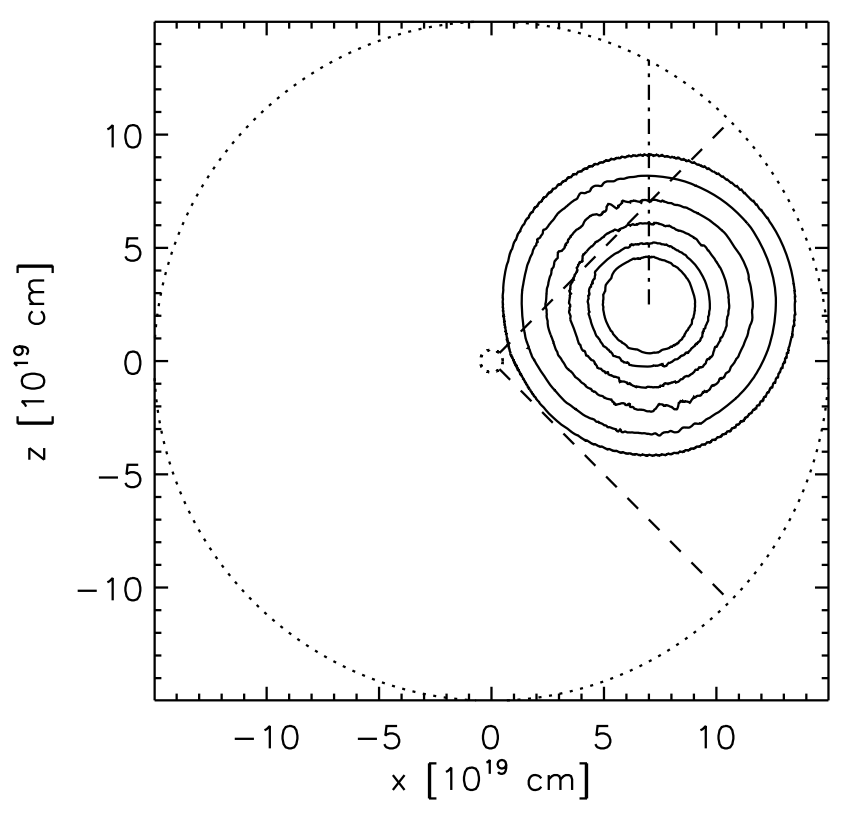

Fig. 7. Lines of constant density in the meridional plane $\phi^{(n)}=0$ obtained from our simulation of a Taylor-Sedov explosion. The snapshot is taken at a simulation time $t_{\text {sim }}=2.0 \times 10^{11} \mathrm{~s}$ which corresponds to an explosion time $t_{\exp } \approx 2.34 \times 10^{11} \mathrm{~s}$. The dashed lines mark the YinYang boundary, while the two dotted circles represent the inner and outer radial boundary of the computational domain, respectively. The data presented in Fig. 6 are re-sampled along the dashed-dotted line.

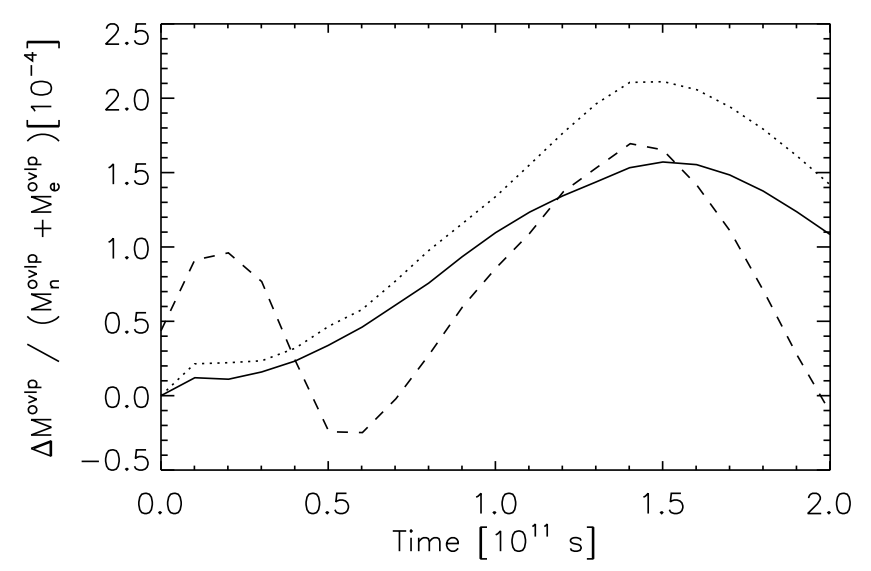

Fig. 8. Evolution of the mass within the overlap region for the TaylorSedov test case computed on the Yin grid, $M_{n}^{\text {ovlp }}$ minus the mass computed on the Yang grid, $M_{\mathrm{e}}^{\text {ovlp }}$, divided by the sum of these two masses. The dashed, dotted and solid lines give the solutions computed on a grid of $400 \times 32 \times 92 \times 2$ zones, (i.e., $3^{\circ}$ angular resolution), $400 \times 92 \times 272 \times 2$ zones (i.e., $1^{\circ}$ angular resolution), and $400 \times 182 \times 542 \times 2$ zones (i.e., $0.5^{\circ}$ angular resolution), respectively.

both considered configurations in the middle and right panels of Fig. 11, respectively. Both errors show a convergence behavior with higher grid resolution, and tend to saturate at large values of $L$. This behavior is similar to what is described in Müller \& Steinmetz (1995). In addition, for lower grid resolution the accuracy saturates at a lower number of spherical harmonics compared to calculations with a higher grid resolution. This is expected since higher order terms in the multipole expansion are not well represented on grids of lower angular resolution.

We also tested our extended Poisson solver discussed in Sect.3.2. In Table 1 we compare the mean errors in the 

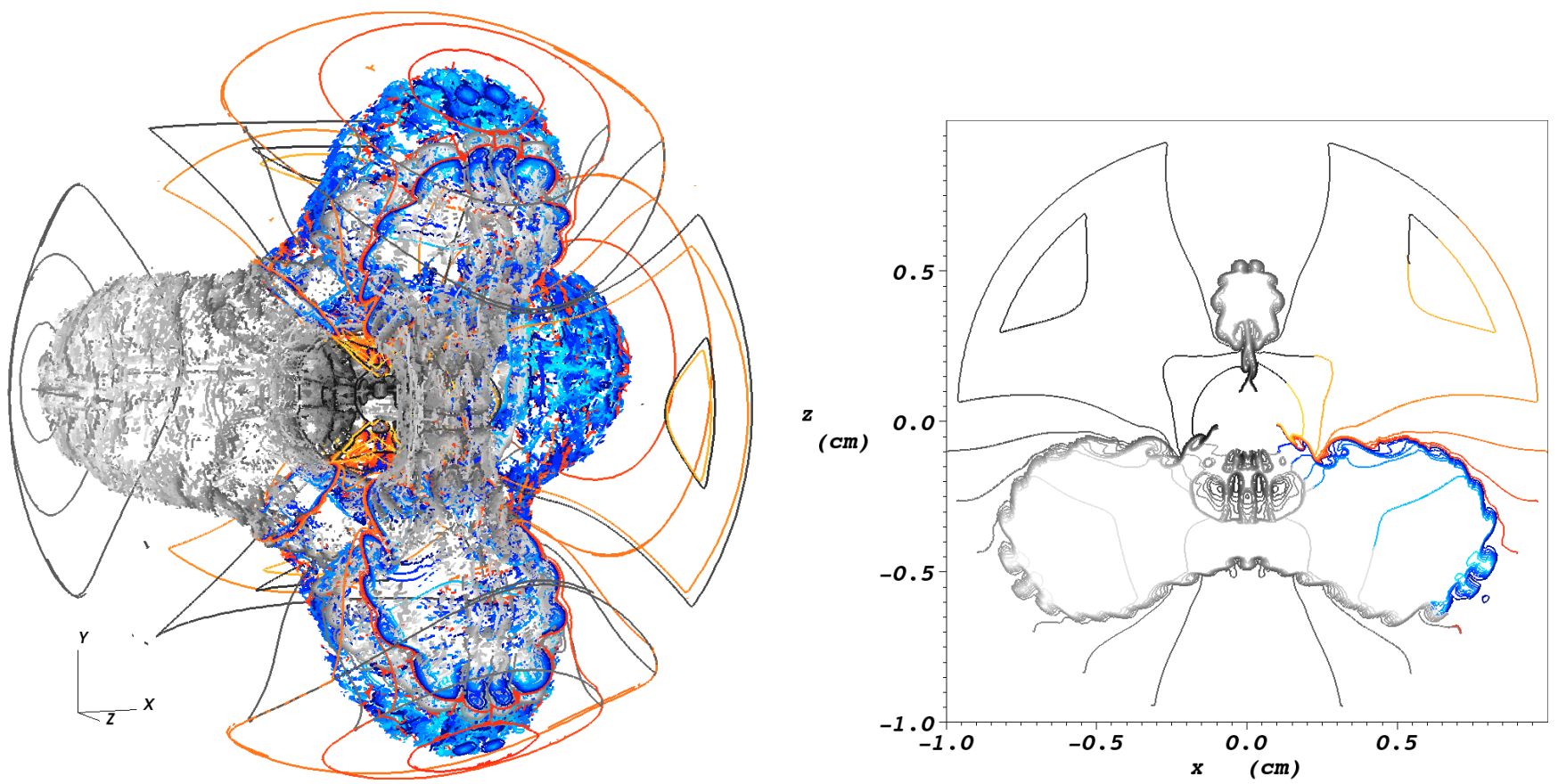

Fig. 9. Surfaces of constant density in 3D (left) and 2D (right; meridional cut at $\left.\phi^{(n)}=0\right)$ resulting from the simulation of the Rayleigh-Taylor instability described in the text at $t=2.85 \mathrm{~s}$. Contour lines on the Yin grid are shown using the blue-yellow colors while contour lines on the Yang grid are displayed using the white-black colors.

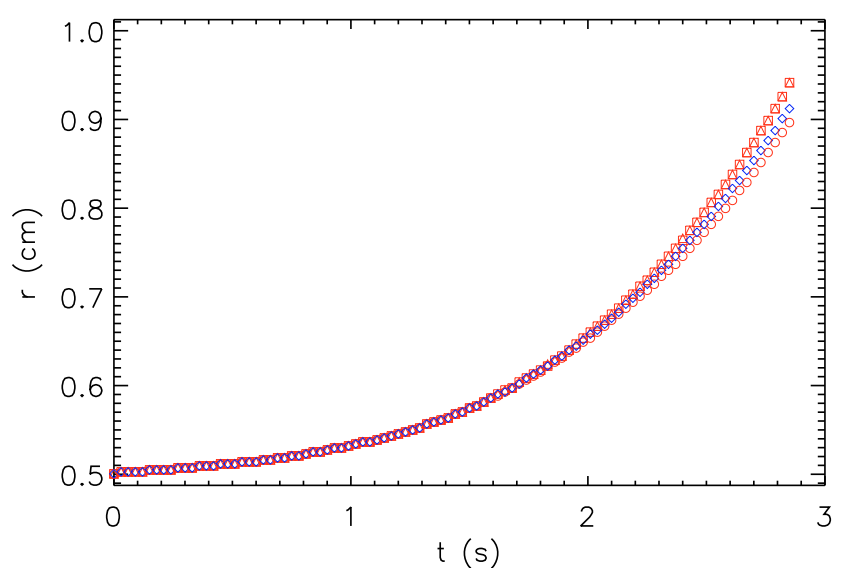

Fig. 10. Position of the heads of the RTI bubbles versus time. Red symbols (circles, triangles, and squares) show data from the Yin grid, while blue symbols (diamonds) represent data on the Yang grid.

components of the gravitational acceleration for both the prolate spheroid and the sphere test case computed with the numerically differentiated gravitational potential given in Eq. (14) with those obtained from the analytic expression given in Eqs. (21), (26), and (27), respectively. We used a grid of $400 \times 92 \times 272 \times 2$ zones and $L=15$ for this comparison.

For the prolate spheroid test case the "analytically" obtained accelerations exhibit a smaller mean error, especially for the $\theta$ - and $\phi$-component of the gravitational acceleration. This results from a strong decrease of the maximum error, which is large in regions where the angular components of the gravitational acceleration approach zero, i.e., near the major and minor axes of the prolate spheroid. However, in these regions the accelerations in $\theta$ and $\phi$-direction are orders of magnitude smaller than the radial component. Thus, they contribute only a tiny fraction to the total acceleration. In the sphere test case both variants of the extended Poisson solver produce similar mean errors. Based on these results we conclude that the extended Poisson solver, which provides the gravitational acceleration using analytic expressions, works properly. Moreover, it gives a slightly more accurate gravitational acceleration, as it does not involve numerically differencing the gravitational potential. Nevertheless, for the reasons stated in Sect. 3.2, we prefer to use the Poisson solver of Müller \& Steinmetz (1995) in our simulations.

\subsection{Self-gravitating polytropes}

Using our Yin-Yang grid based hydro-code we have also considered self-gravitating, non-rotating and rotating equilibrium polytropes. Both kinds of polytropes provide another test of the Poisson solver, and a test of how well our hydrodynamics code can keep a self-gravitating configuration in hydrostatic and stationary equilibrium, respectively. In addition, the rotating polytrope also serves to test the proper working of the Yin-Yang boundary treatment, as it involves a considerable and systematic flow of mass, momentum and energy flux across that boundary due to the polytrope's rotation.

The polytropes have a polytropic index $n=1$, a polytropic constant $\kappa=1.455 \times 10^{5}$, and a central density of $\rho_{\mathrm{c}}=$ $7.905 \times 10^{14} \mathrm{~g} / \mathrm{cm}^{3}$. For our test runs we interpolated equilibrium polytropes calculated with the method of Eriguchi \& Müller (1985) onto a Yin-Yang sphere, and simulated their dynamic evolution (occurring as the interpolated configuration is not in perfect hydrostatic equilibrium). The central region $(r<1 \mathrm{~km})$ of the polytrope is cut out and replaced by a corresponding point mass to allow for a larger time step.

We use an artificial atmosphere technique to handle those regions of the computational grid that lie outside the (rotating, i.e., non-spherical) polytrope. The density in the atmosphere is set equal to a value $\rho_{\mathrm{atm}}=10^{-10} \rho_{\mathrm{c}}$, where $\rho_{\mathrm{c}}$ is the central density of the polytrope. Here, atmosphere denotes any grid zone 


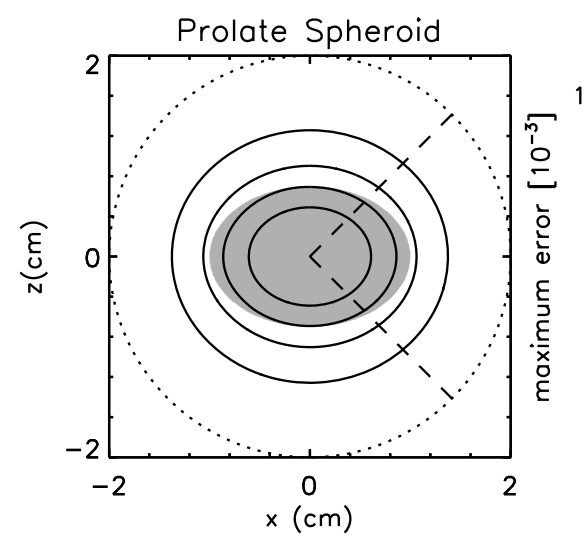

Maximum error spheroids
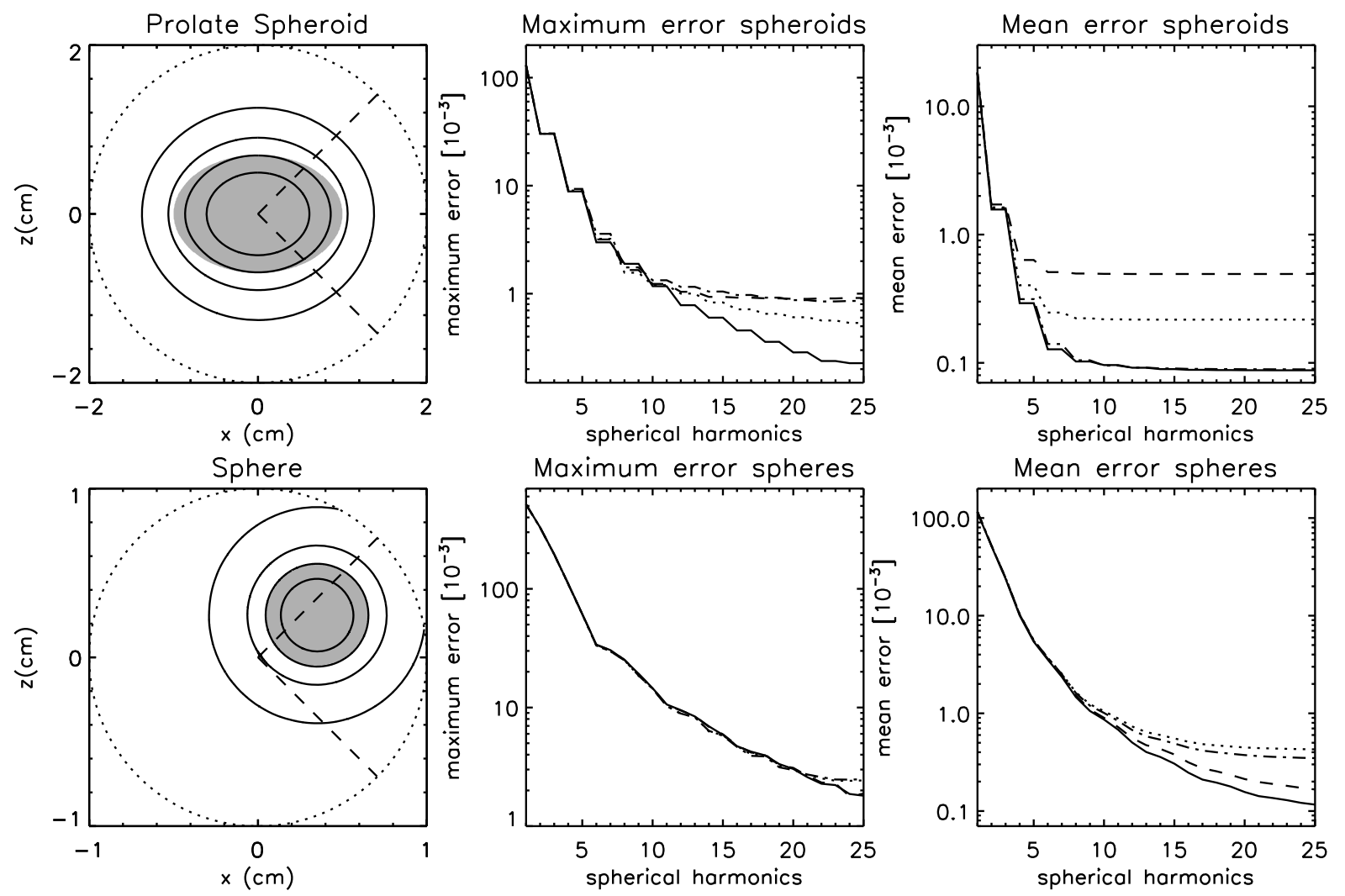

Fig. 11. Contour lines of the gravitational potential (left column) for two homogeneous self-gravitating configurations: a prolate spheroid (top row) with an axis ratio of 0.7, and a sphere (bottom row). The configurations are indicated by the dark-gray shaded areas. Dashed lines show the Yin-Yang boundary, while dotted lines indicate the outer radial boundary of the computational grid. The middle and right columns give the maximum and mean error of the numerically calculated gravitational potential for different grid resolutions as a function of the number of spherical harmonics used in our multipole gravity solver. The solid, dotted, dashed, and dashed-dotted lines in both columns correspond to a grid resolution of $400 \times 92 \times 272 \times 2$ zones, $400 \times 47 \times 137 \times 2$ zones, $200 \times 92 \times 272 \times 2$ zones, and $200 \times 47 \times 137 \times 2$ zones, respectively.

Table 1. Mean errors in the gravitational acceleration.

\begin{tabular}{ccccc}
\hline \hline \multirow{2}{*}{ Component } & \multicolumn{2}{c}{ Prolate spheroid } & \multicolumn{2}{c}{ Sphere } \\
& Poisson solver & extended Poisson solver & Poisson solver & extended Poisson solver \\
\hline$\hat{\mathbf{r}}$ & $4.821 \times 10^{-4}$ & $4.698 \times 10^{-4}$ & $1.598 \times 10^{-2}$ & $1.557 \times 10^{-2}$ \\
$\hat{\theta}$ & $6.134 \times 10^{-2}$ & $2.592 \times 10^{-2}$ & $1.67 \times 10^{-2}$ & $1.67 \times 10^{-2}$ \\
$\hat{\phi}$ & $1.245 \times 10^{-2}$ & $2.435 \times 10^{-3}$ & $1.655 \times 10^{-2}$ & $1.655 \times 10^{-2}$ \\
\hline
\end{tabular}

whose density is less than the cut-off density $\rho_{\text {cut-off }}=10^{-7} \rho_{\max }$. Furthermore, for all zones in the atmosphere the velocity is set to zero in order to keep the atmosphere quiet. This procedure is applied at the end of every time step throughout the simulation. A zero-gradient boundary condition is imposed at the outer radial boundary, and a reflecting boundary condition at the inner one. The polytrope's evolution is followed for $10 \mathrm{~ms}$ corresponding to approximately 10 dynamic time scales in order to check how well the initial approximate equilibrium configuration is maintained by the Yin-Yang code.

For the non-rotating polytrope, we employ a grid of $400 \times$ $20 \times 56 \times 2$ zones. Note that we are able to use a relatively low angular resolution compared to the other tests, because the problem has spherical symmetry. Our results show that the polytrope stays perfectly spherically symmetric throughout the simulation, and that the non-radial velocities inside the polytrope remain zero. This demonstrates that the Yin-Yang grid is able to preserve the initial spherical symmetry. Figure 12 shows the evolution of the central density (more precisely of the density of the innermost radial zone at $r=1 \mathrm{~km}$ ), which exhibits oscillations with an amplitude of the order of $10^{-4}$ without any sign of a systematic trend. Comparing the initial radial distributions of the density (Fig. 13, upper panel) and the radial velocity (Fig. 13, lower panel) of the polytrope with those after $10 \mathrm{~ms}$ of evolution, we find no significant deviations. Relative changes in the density profile are of the order of $10^{-4}$, comparable to the size of the fluctuations of the central density. Only for zones near the edge of the polytrope the deviations can reach a level of up to $20 \%$, in particular in the zone next to the atmosphere. The figure also shows that data points from the Yin and the Yang grid lie on top of each other confirming that the code preserves the initial spherical symmetry of the polytrope very well. Except for the zones at the polytrope's surface, where the radial velocity is fluctuating at a level of approximately $2 \times 10^{8} \mathrm{~cm} / \mathrm{s}$, the radial velocities are less than $10^{6} \mathrm{~cm} / \mathrm{s}$ (i.e., less than $0.1 \%$ of the local sound speed). Thus, we conclude that a non-rotating ( $n=1)$ equilibrium polytrope is correctly handled by our YinYang hydro-code.

The rotating polytrope needs a higher grid resolution in $\theta$-direction, as it is no longer spherically symmetric. Thus, we 
A. Wongwathanarat et al.: An axis-free overset grid in spherical polar coordinates for simulating 3D self-gravitating flows

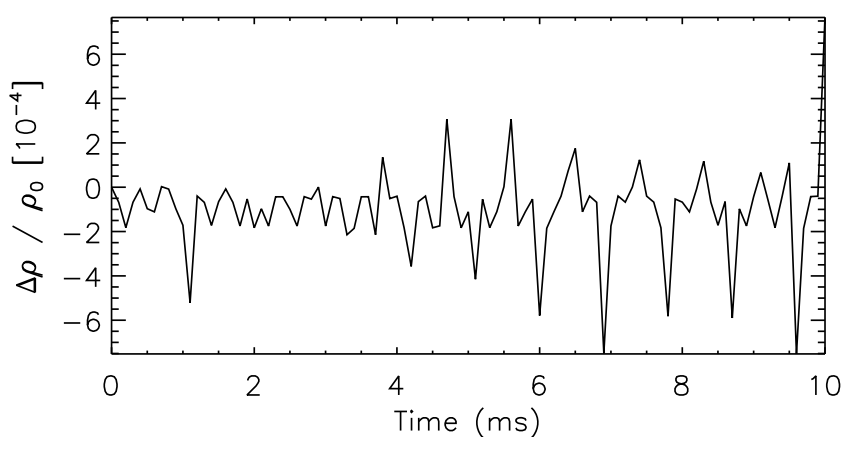

Fig. 12. Relative change of the central density of a non-rotating (nearly) equilibrium polytrope as a function of time.

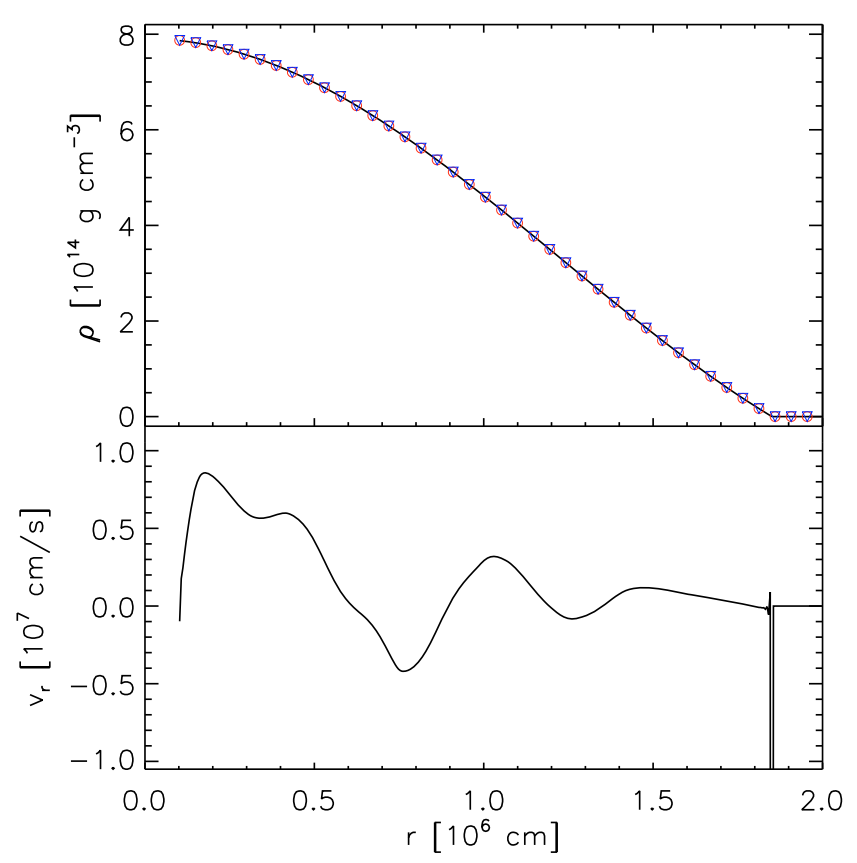

Fig. 13. Density (top) and radial velocity (bottom) of a non-rotating $n=1$ equilibrium polytrope as a function of radius after $t=10 \mathrm{~ms}$ of "evolution". In the top panel, the solid line shows the initial density profile. Red circles and blue triangles correspond to data from the Yin and the Yang grid, respectively.

used a grid resolution of $400 \times 92 \times 272 \times 2$ zones for this simulation. The initial oblate equilibrium configuration has an axis ratio of 0.7 . We, again, evolve the configuration for $10 \mathrm{~ms}$ to test the correct treatment of the situation by our Yin-Yang hydro-code.

Figure 14 shows the relative variation of the central density as a function of time along an equatorial ray $\left(\theta^{(n)}=\pi / 2\right.$; $\left.\phi^{(n)}=0\right)$ and along the pole $\left(\theta^{(n)}=0\right)$, respectively. One also recognizes a slight systematic trend in the behavior of the density fluctuation, which is steeper along the equator than at the pole. However, in both cases the relative increase of the central density is very small $\left(\sim 10^{-3}\right)$. The initial radial density profiles along the pole and the equator do not show any significant change during the $10 \mathrm{~ms}$ of evolution we have simulated with the Yin-Yang code (Fig. 15, upper panel). The axis ratio has slightly increased to a value of 0.719 . The radial velocities (Fig. 15, lower panel) are larger than in the non-rotating case by about an order of magnitude, because it is obviously more difficult to keep a rotating polytrope in equilibrium than a nonrotating (spherically symmetric) one. We again find the largest

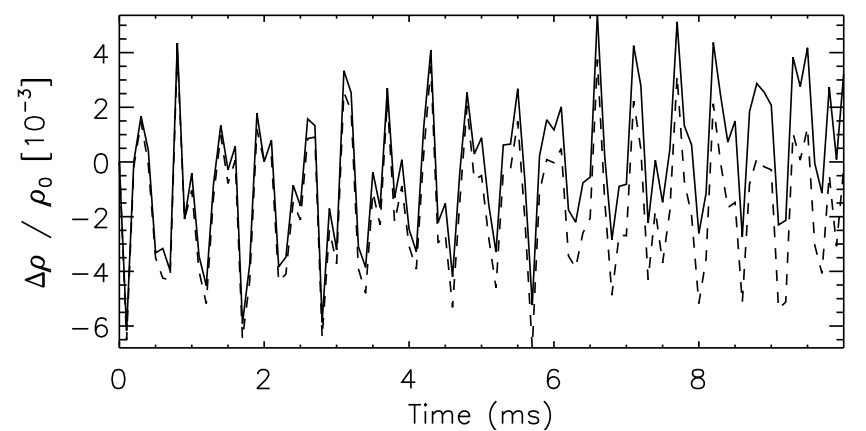

Fig. 14. Same as Fig. 12 but for a rotating polytrope. The solid and dashed curves show the relative variation of the density along an equatorial ray $\left(\theta^{(n)}=\pi / 2 ; \phi^{(n)}=0\right)$ and along the pole $\left(\theta^{(n)}=0\right)$, respectively.

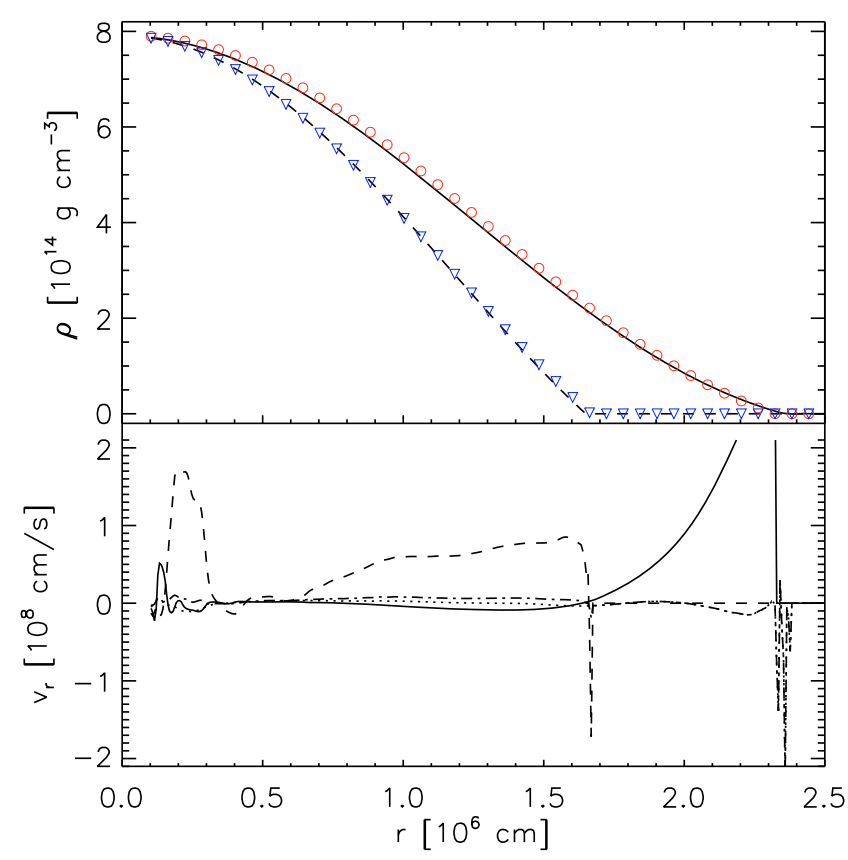

Fig. 15. Density (upper panel) and radial velocity (lower panel) of a $n=1$ rotating polytrope in stationary equilibrium as a function of radius after $t=10 \mathrm{~ms}$ of "evolution". In both panels the solid and dashed lines show the profiles along an equatorial ray $\left(\theta^{(n)}=\pi / 2, \phi^{(n)}=0\right)$ and along the pole $\left(\theta^{(n)}=0\right)$, respectively. Red circles and blue triangles in the upper panel correspond to data from the Yin and the Yang grid, respectively. In the lower panel, we show in addition time averaged (over the interval $t=[9,10] \mathrm{ms}$ ) velocity profiles along the equatorial ray (dotted) and the pole (dashed-dotted).

radial velocities (a few times $10^{8} \mathrm{~cm} / \mathrm{s}$ ) near the surface of the polytrope, especially along the equator. However, these velocities vary with time. When averaged over time (in the time interval $t=[9,10] \mathrm{ms}$ ) the profiles become flatter and the velocities smaller. This confirms that the polytrope is oscillating around its equilibrium configuration.

\section{Conservation problem}

The Yin-Yang grid has a disadvantage common with other types of overlapping grids (see, e.g., Chesshire \& Henshaw 1994; Wang 1995; Wu et al. 2007). The communication via interpolation between the two grid patches does not guarantee conservation of conserved quantities even though the finitevolume difference scheme employed on each grid patch is conservative. Non-conservation occurs when a flow across the YinYang boundary is present. This is the case in most of our tests 


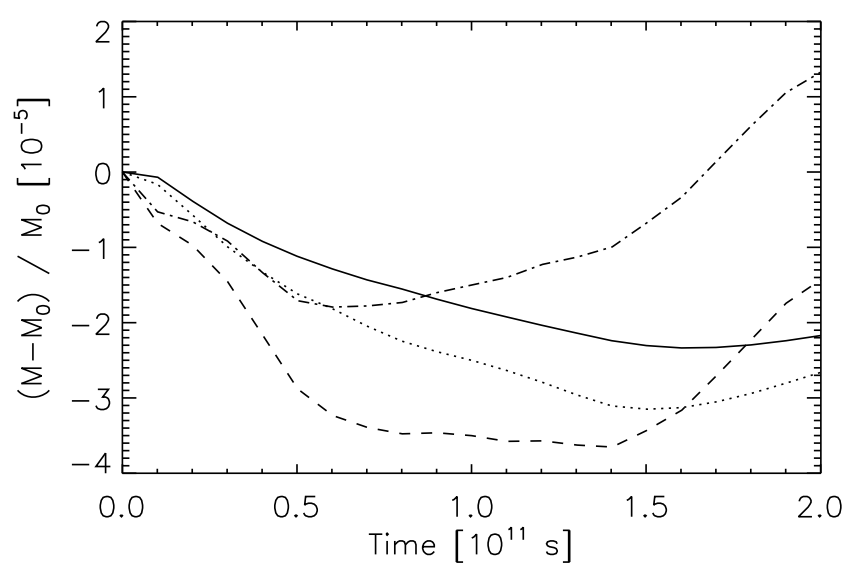

Fig. 16. Evolution of the relative mass loss, $\left(M-M_{0}\right) / M_{0}$, where $M_{0}$ is the initial total mass, for the Taylor-Sedov test simulated on three different (angular) grids with $400 \times 32 \times 92 \times 2$ zones (i.e., $3^{\circ}$ angular resolution; dashed line), $400 \times 92 \times 272 \times 2$ zones (i.e., $1^{\circ}$ angular resolution; dotted line), and $400 \times 182 \times 542 \times 2$ zones (i.e., $0.5^{\circ}$ angular resolution; solid line), respectively.

except for the simulation of the non-rotating polytrope that involves only radial flow.

Nevertheless, we are still able to obtain sufficiently good results for all the test simulations discussed in the previous section. The degree of non-conservation is highly problem dependent. A simulation involving a considerable and systematic flow across the Yin-Yang boundary, as e.g., in the case of the rotating polytrope, will result in a larger degree of non-conservation. We observe that the total mass increases by $0.07 \%$ within $10 \mathrm{~ms}$ (or about ten dynamical timescales) in the case of the rotating polytrope. For the Taylor-Sedov test case, which is the cleanest test case in this respect (as it involves, e.g., no boundary effects like the shock tube, and e.g., no artificial atmosphere like the rotating polytrope), we find a mass loss of the order of $10^{-5}$, only. As Fig. 16 demonstrates this mass loss can be reduced by using a higher angular resolution.

Conservation of conserved scalar quantities can be obtained to machine precision by applying the algorithm described in detail in Peng et al. (2006), and summarized below. According to this algorithm scalar fluxes at the outer edges of boundary zones of both the Yin and the Yang grid are replaced by scalar fluxes computed using only "interior" fluxes from adjacent grid zones.

As an illustration, consider the Yin-Yang grid overlap configuration in Fig. 17, where PQRS is a grid zone at the boundary of the Yang grid (blue) which overlaps with the underlying grid zone ABCD of the Yin grid (red). Fluxes referring to the Yin and the Yang grid are denoted by $f$ and $g$, respectively. flux

The boundary flux $g_{\mathrm{PQ}}$ of the Yang grid is replaced by the

$f_{\mathrm{PQ}}=f_{\mathrm{FQ}}+f_{\mathrm{PF}}$,

where $f_{\mathrm{FQ}}$ and $f_{\mathrm{PF}}$ are the fluxes through the segments $\overline{\mathrm{FQ}}$ and $\overline{\mathrm{PF}}$, respectively.

The flux $f_{\mathrm{FQ}}$ in Eq. (33) is calculated using information from zone $\mathrm{ABCD}$. The evolution of a scalar quantity $\xi_{\mathrm{ABCD}}$ of zone $\mathrm{ABCD}$ is given by

$\xi_{\mathrm{ABCD}}^{t+\Delta t}=\xi_{\mathrm{ABCD}}^{t}+\left(f_{\mathrm{AB}}-f_{\mathrm{CD}}+f_{\mathrm{BC}}-f_{\mathrm{AD}}\right)$.
Similarly, for the fraction of the zone ABCD defined by the polygon ABFED one has,

$\xi_{\mathrm{ABFED}}^{t+\Delta t}=\xi_{\mathrm{ABFED}}^{t}+\left(f_{\mathrm{AB}}-f_{\mathrm{CD}} \frac{\overline{\overline{\mathrm{DE}}}}{\overline{\mathrm{CD}}}+f_{\mathrm{BC}} \frac{\overline{\mathrm{BF}}}{\overline{\mathrm{BC}}}-f_{\mathrm{AD}}-f_{\mathrm{EF}}\right)$.

Assuming a piecewise constant state within the zone ABCD, Eqs. (34) and (35) lead to

$\alpha\left(\xi_{\mathrm{ABCD}}^{t+\Delta t}-\xi_{\mathrm{ABCD}}^{t}\right)=\xi_{\mathrm{ABFED}}^{t+\Delta t}-\xi_{\mathrm{ABFED}}^{t}$

where $\alpha$ is the overlapping volume fraction (area) described in Sect. 3. Therefore,

$\alpha\left(f_{\mathrm{AB}}-f_{\mathrm{CD}}+f_{\mathrm{BC}}-f_{\mathrm{AD}}\right)=f_{\mathrm{AB}}-f_{\mathrm{CD}} \frac{\overline{\overline{\mathrm{DE}}}}{\overline{\mathrm{CD}}}+f_{\mathrm{BC}} \frac{\overline{\mathrm{BF}}}{\overline{\mathrm{BC}}}-f_{\mathrm{AD}}-f_{\mathrm{EF}}$.

Note that the flux $f_{\mathrm{EF}}$ is the only unknown in Eq. (37). Since the intersection points $E$ and $F$ are already known from the step to calculate the volume fraction $\alpha$, the lengths of all segments can be obtained. The flux $f_{\mathrm{FQ}}$ is then given by

$f_{\mathrm{FQ}}=f_{\mathrm{EF}} \frac{\overline{\mathrm{FQ}}}{\overline{\mathrm{EF}}}$.

After obtaining the still missing flux $f_{\mathrm{PF}}$ in Eq. (33) by a similar procedure, the scalar quantity $\xi_{\mathrm{PQRS}}$ of the boundary zone PQRS is updated according to

$\xi_{\mathrm{PQRS}}^{t+\Delta t}=\xi_{\mathrm{PQRS}}^{t}+\left(g_{\mathrm{QR}}-g_{\mathrm{PS}}+g_{\mathrm{RS}}-f_{\mathrm{PQ}}\right)$.

This procedure is then repeated to update all boundary grid zones.

After implementing the above algorithm we are able to conserve mass and total energy up to machine precision. However, the conservation of momentum is more complicated since the momentum equations in spherical coordinates involve not only flux (i.e., divergence) terms but also source terms (due to the presence of fictitious and pressure forces), and due to the "mixing" of momentum components as the Yin and Yang grid patches are rotated relative to each other (see Fig. 17).

As we have not yet devised and implemented a corresponding momentum conservation algorithm, momentum is not yet perfectly conserved in our code. For that reason we also refrain from using the scalar conservation algorithm described above, since in some simulations (e.g., in the Taylor-Sedov explosion simulation) we encountered a negative internal energy in some zones due to the inconsistency arising from the perfect conservation of mass and total energy on one hand and the imperfect conservation of momentum on the other hand. In our test runs the momentum violation is small, e.g., amounting to $0.24 \%(0.03 \%)$ angular momentum loss in the case of the rotating polytrope for a grid with three (one) degree angular resolution.

\section{Performance and efficiency}

One of the main purposes in implementing the Yin-Yang grid is to ease the severe restriction imposed on the size of the time step for any explicit hydrodynamics scheme by the CFL condition in the polar regions of 3D simulations using a grid in spherical polar coordinates. In most applications the size of the time step is restricted most strongly by the size of the zones in $\phi$-direction, which is smaller than the size in $\theta$-direction by the factor $\sin \theta$ assuming an equal angular resolution $\delta \equiv \Delta \theta=\Delta \phi$ in both angular directions. 


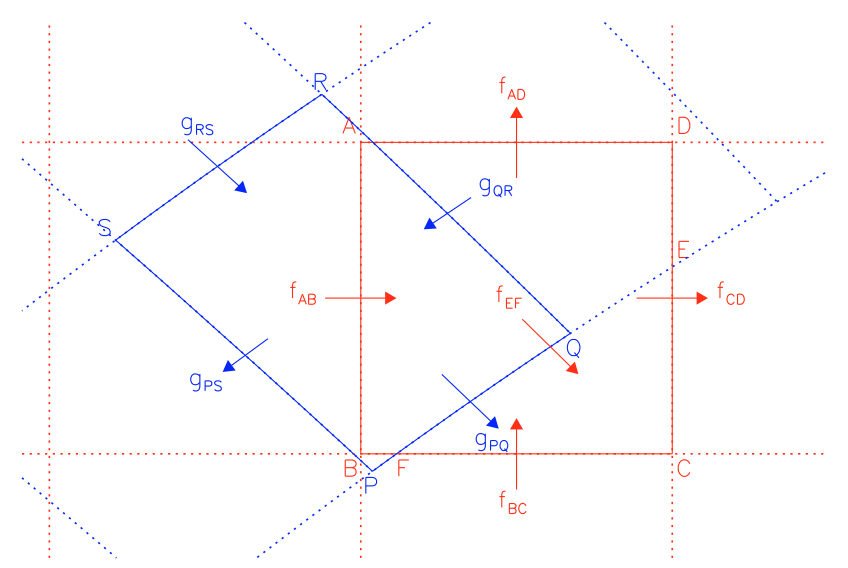

Fig. 17. Illustration of the Yin-Yang grid overlap configuration, where PQRS is a grid zone at the boundary of the Yang grid (blue) which overlaps with the underlying grid zone $A B C D$ of the Yin grid (red). Fluxes referring to the Yin and the Yang grid are denoted by $f$ and $g$, respectively.

Table 2. Expected gain factor when using the Yin-Yang grid.

\begin{tabular}{lcc}
\hline \hline Computational domain & Angular grid resolution & Gain factor \\
\hline full $4 \pi$ sphere & $3^{\circ}$ & 26 \\
& $2^{\circ}$ & 40 \\
& $1^{\circ}$ & 80 \\
$\begin{array}{l}\text { sphere except for a cone } \\
\text { of } 5^{\circ} \text { half opening angle } \\
\text { cut-out at both poles }\end{array}$ & $1^{\circ}$ & 7 \\
\hline
\end{tabular}

For a spherical polar grid the factor $\sin \theta$ implies (assuming zone centered variables) a minimum zone size

$d_{\phi}^{\mathrm{sph}} \equiv \delta \sin (\delta / 2)$

(in radians) in $\phi$-direction for the first zone next to the pole. Typically, $\sin (\delta / 2) \approx 10^{-2}$. On the other hand, applying the YinYang grid yields

$d_{\phi}^{Y Y} \equiv \delta \sin (\pi / 4-\delta / 2)$

for the size of the smallest zone in $\phi$-direction, which is typically about 0.7 . Hence, for the Yin-Yang grid the smallest zone size in azimuthal direction is larger by the ratio

$\frac{d_{\phi}^{Y Y}}{d_{\phi}^{\mathrm{sh}}}=\frac{\sin (\pi / 4-\delta / 2)}{\sin (\delta / 2)}$

compared to the spherical polar grid.

Table 2 gives the value of this ratio for grids of various angular resolution, and various computational domains. These numbers provide an estimate of the gain in computation time one can expect when using the Yin-Yang grid instead of the spherical polar grid.

However, the gain factor calculated from the relative grid spacings does not determine the gain in the size of the time step, as the latter is given in a more complicated way by the CFL condition

$$
\begin{aligned}
\Delta t_{\mathrm{CFL}}<C\left(\left|\frac{v_{r}}{\Delta r}\right|\right. & +\left|\frac{v_{\theta}}{r \Delta \theta}\right|+\left|\frac{v_{\phi}}{r \sin \theta \Delta \phi}\right| \\
& \left.+\sqrt{\frac{c_{s}^{2}}{\Delta r^{2}+(r \Delta \theta)^{2}+(r \sin \theta \Delta \phi)^{2}}}\right)^{-1 / 2},
\end{aligned}
$$

where $C, v_{r}, v_{\theta}, v_{\phi}$, and $c_{s}$ are the Courant factor, the flow velocities in radial, colatitude and azimuthal direction, and the local sound speed, respectively. The CFL condition shows that the increase in the size of the CFL time step is somewhat smaller than implied by the gain factor resulting from the ratio of the sizes of the smallest zones of the Yin-Yang grid and the spherical polar grid. In addition, the increase of the time step is problem dependent.

Besides the performance gain due to the increased size of the CFL time step, the Yin-Yang grid also requires less computational zones to cover the full sphere, and thus less computational time. For an angular resolution $\delta$ the spherical polar grid needs

$(\pi / \delta) \times(2 \pi / \delta)$

zones to cover the full sphere, while the Yin-Yang grid requires only

$(\pi / 2 \delta+2) \times(3 \pi / 2 \delta+2) \times 2$

zones. Hence, up to $25 \%$ fewer computational zones are required. The gain depends only weakly on angular resolution and is problem independent.

However, employing the Yin-Yang grid also requires some extra amount of computation compared to the spherical polar grid (see Sect. 3). In the following we only consider the extra costs of calculations during the actual simulation, but not the extra costs arising during the initialization, since these are negligible. We emphasize again that there are two major extra sets of calculations necessary when applying the Yin-Yang grid. The first set concerns the interpolation of the ghost zone values that are needed for the communication between the Yin and Yang grid patches. The second set arises from the interpolation of the density onto the auxiliary spherical polar grid grid and the interpolation of the gravitational potential back from the auxiliary grid onto the Yin-Yang grid. Exploiting the algorithms described in Sect. 3, the computational cost for both parts is almost negligible compared to the total computing time. Interpolation of the ghost zone values requires only $2.3 \%$ of the total computing time per cycle in simulations with self-gravity, while the interpolation of density and gravitational potential performed within the gravity solver accounts for $1.5 \%$ of the computing time needed for the gravity solver. This corresponds to approximately $0.3 \%$ of the computing time per cycle.

To obtain actual numbers for the gain, we performed several timing tests including simulations with and without self-gravity using four different grid resolutions. The tests were carried on an IBM Power6 using a single processor. According to these tests the computing time per cycle for the Yin-Yang grid averaged over five cycles is approximately $15 \%$ and $20 \%$ smaller than for the spherical polar grid for simulations without selfgravity and with $2^{\circ}$ and $1^{\circ}$ angular resolution, respectively. For simulations including self-gravity, the gain factor decreases by $3 \%$ approximately.

Concerning the gain from the less restrictive CFL condition, we consider the case of the rotating polytrope since the size of the time step does not vary much throughout the simulation. For an angular resolution of $1^{\circ}$, we find a gain of approximately a factor of 63 when using the same Courant number both for the Yin-Yang grid and the spherical polar grid.

\section{Conclusion}

A two-patch overset grid in spherical coordinates called the "Yin-Yang" grid is successfully implemented into our 
3D Eulerian explicit hydrodynamics code, PROMETHEUS, including in particular the treatment of self-gravitating flows. The Yin-Yang grid eases the severe restriction of the time step size in the polar regions of the sphere, because each Yin-Yang grid patch contains only the low-latitude part of the usual spherical polar grid. From our experiences, the implementation steps are easy and straightforward for a hydrodynamics code using directional splitting and having 3D spherical polar coordinates already implemented due to the simplicity of the Yin-Yang transformation and its symmetry property. Basically it involves doubling the state variable arrays, calling the 1D core hydrodynamics solver in angular directions for both the Yin and the Yang grid, and adding a subroutine that handles the Yin-Yang transformation and the interpolation of variables between both grids.

We validated the code with several standard hydrodynamic tests. The test results show good agreement with analytic solutions if these are available. Furthermore, as demonstrated by three of our test problems - a planar shock crossing the YinYang grid boundary, an off-center Taylor-Sedov explosion involving mass, momentum (all three components) and energy flux across the Yin-Yang grid boundary, and a polytrope whose rotation leads to considerable and systematic mass, momentum (only angular components) and energy flux across the Yin-Yang grid boundary - the Yin-Yang grid does not introduce any numerical artifact at the internal Yin-Yang boundary. The tests also confirm that the numerical solutions obtained with the Yin-Yang grid do not show any evidence of a preferred radial direction, as it eliminates numerical axes artifacts which seriously flaw the flow near the coordinate symmetry axis when using a spherical polar grid. Besides successfully simulating a Taylor-Sedov explosion and self-gravitating (rotating and non-rotating) equilibrium polytropes the code has also passed another astrophysically relevant test involving the growth of Rayleigh-Taylor instabilities.

Because the communication between the two grid patches involves interpolation, flows across the Yin-Yang boundary cause some small amount of non-conservation of conserved quantities. However, even for the (in this respect) severe test case of the rotating polytrope involving large flows across the Yin-Yang boundary, we observe only a small amount (less than one percent) of non-conservation.

The Yin-Yang grid offers a large gain in computing time arising from two sources. Firstly, the number of computational zones needed is reduced by $20 \%$ approximately depending on the angular resolution. This gain reduces the computing time per cycle and is problem independent. Secondly, the size of the CFL time step is considerably enhanced, because the polar regions with converging meridional coordinate lines are not present in case of the Yin-Yang grid. The corresponding gain in time step size highly depends on the problem simulated. The extra costs for interpolation between the two grid patches and the interpolation performed in the gravity solver are negligible compared to the gain in the time step size.

In conclusion, our implementation of the Yin-Yang grid into the multi-dimensional hydrodynamics code PROMETHEUS brings about the possibility to simulate three dimensional selfgravitating hydrodynamic flows in spherical coordinates which, in most cases, have been computationally inaccessible up to now due to the prohibitively large computational costs. With the possibility to add more physics such as neutrino transport (work in progress), the new code version can be used to carry out, e.g., core collapse supernova simulations in 3D.

Acknowledgements. This research was supported by the Deutsche Forschungsgemeinschaft through the Transregional Collaborative Research Centers SFB/TR 27 "Neutrinos and Beyond" and SFB/TR 7 "Gravitational Wave Astronomy", and the Cluster of Excellence EXC 153 "Origin and Structure of the Universe". The simulations were performed at the Rechenzentrum Garching (RZG) of the Max-Planck-Society. We would like to thank Prof. A. Kageyama for sharing with us his Yin-Yang interpolation routine for scalar/vector fields, and the anonymous referee for his/her useful and supportive comments.

\section{References}

Blondin, J. M., \& Mezzacappa, A. 2006, ApJ, 642, 401

Chandrasekhar, S. 1969, Ellipsoidal figures of equilibrium (Yale Univ. Press)

Chesshire, G., \& Henshaw, W. 1994, SIAM J. Sci. Comput., 15, 819

Collela, P., \& Woodward, P. R. 1984, J. Comput. Phys., 54, 174

Eriguchi, Y., \& Müller, E. 1985, A\&A, 147, 161

Fragile, P. C., Lindner, C. C., Anninos, P., \& Salmonson, J. D. 2009, ApJ, 691, 482

Iwakami, W., Kotake, K., Ohnishi, N., Yamada, S., \& Sawada, K. 2008, ApJ, 678,1207

Janka, H.-T., Scheck, L., Kifonidis, K., Müller, E., \& Plewa, T. 2005, in The Fate of the Most Massive Stars, ed. R. Humphreys, \& K. Stanek, ASP Conf. Ser., 332,363

Kageyama, A., \& Sato, T. 2004, Geochem. Geophys. Geosyst., 5

Kifonidis, K., Plewa, T., Janka, H.-T., \& Müller, E. 2003, A\&A, 408, 621

Koldoba, A. V., Romanova, M. M., Ustyugova, G. V., \& Lovelace, R. V. E. 2002 , ApJ, 576, L53

Landau, L., \& Lifshitz, E. 1959, Fluid mechanics, 6 (Pergamon)

Mezzacappa, A., Bruenn, S., Blondin, J. M., Hix, W., \& Messer, O. 2006, in AIP Conf. Proc., 924, 234

Müller, E., \& Steinmetz, M. 1995, Comput. Phys. Commun., 89, 45

Ohnishi, N., Kotake, K., \& Yamada, S. 2007, ApJ, 667, 375

Peng, X., Xiao, F., \& Takahashi, K. 2006, Q.J.R. Meteorol. Soc., 132, 979

Romanova, M. M., Ustyugova, G. V., Koldoba, A. V., Wick, J. V., \& Lovelace, R. V. E. 2003, ApJ, 595, 1009

Ronchi, C., Iacono, R., \& Paolucci, P. S. 1996, J. Comput. Phys., 124, 93

Scheck, L. 2006, Ph.D. Thesis, Technical University Munich

Scheck, L., Kifonidis, K., Janka, H.-T., \& Müller, E. 2006, A\&A, 457, 963

Sod, G. A. 1978, J. Comput. Phys., 27, 1

Toro, E. F. 1997, Riemann solvers and numerical methods for fluid dynamics a practical introduction (Springer)

Wang, Z. 1995, J. Comput. Phys., 122, 96

Wu, Z.-N., Xu, S.-S., Gao, B., \& Zhuang, L.-S. 2007, Comput. Fluids, 36, 1657 Zink, B., Schnetter, E., \& Tiglio, M. 2008, Phys. Rev. D, 77 